\title{
Die sprachlich-diskursive Darstellung komplexer Zusammenhänge im Fachunterricht
}

\section{Vertextungsverfahren des Erklärens und Argumentierens}

\begin{abstract}
Ziel dieses Beitrags ist es, Vertextungsverfahren des Erklärens und Argumentierens in fachlichen Unterrichtsgesprächen zu beschreiben und auf diesem Wege die Sprachlichkeit fachlicher Erkenntnis- und Lernprozesse genauer zu fassen. Vertextungsverfahren werden als sozial etablierte und kulturell sedimentierte Praktiken aufgefasst, mit denen komplexe Sachverhalte unter Bedingungen der Interaktivität sukzessive in geordneter Weise her- und dargestellt werden. Sie zeichnen sich durch unterschiedliche Weisen des Entpackens bzw. Zusammenschnürens von Wissenselementen aus und verlangen unterschiedliche Konfigurationen multimodaler Ressourcen. Auf Basis eines Korpus von 140 videographierten Unterrichtsstunden in den Fächern Mathematik und Deutsch werden erstens ausgewählte Vertextungsverfahren exemplarisch beschrieben. Zweitens wird gezeigt, dass Vertextungsverfahren in zwei grundlegend unterschiedlichen Darstellungsmodi operieren, dem Modus des Situierens oder dem Modus des Abstrahierens. Beide Modi erfüllen im Rahmen der (unterrichtlichen) Wissenskonstruktion und -vermittlung distinkte epistemische Funktionen.
\end{abstract}

Keywords: Erklären, Argumentieren, Vertextung, Vertextungsverfahren, Wissen, Unterrichtsinteraktion, Fachunterricht

\section{Sprachlich-diskursive Anforderungen der Konstruktion fachlichen Wissens}

Dass Wissen nicht zuallererst ein im Kopf zu verortendes mentales Phänomen ist, sondern im Gespräch interaktiv erzeugt wird, gehört zu den Grundannahmen der Anthropologie der Wissenschaft (Knorr-Cetina 1984, 2002; Latour \& Woolgar 1986), der Wissenssoziologie (Luckmann 1986) und der ethnomethodo-

Vivien Heller, Bergische Universität Wuppertal, Gaußstraße 20, 42119 Wuppertal, vheller@uni-wuppertal.de

Ә Open Access. ( 2021 Vivien Heller, publiziert von De Gruyter. (c) BY-NC-ND Dieses Werk ist lizensiert unter einer Creative Commons Namensnennung - Nicht-kommerziell - Keine Bearbeitung 4.0 International Lizenz. https://doi.org/10.1515/9783110707168-011 
logischen Konversationsanalyse (u. a. Bergmann \& Quasthoff 2010; Kesselheim 2010; Stivers, Mondada \& Steensig 2011; Goodwin 2013; Janich \& Birkner 2015; Deppermann 2015). Knorr-Cetina (1984: 22) spricht von diskursiven Fabrikationsprozessen, in denen durch „praktisches Räsonieren“ Wissen hergestellt wird. Aussagen erhalten demnach den Status von ,Wissen“ erst dann, wenn sie als Wissensbehauptungen formuliert und von den Interaktionsbeteiligten als gültig ratifiziert werden. So gesehen ist ,Wissen“ das von den Beteiligten „festgestellte und insofern auch beobachtbare Ergebnis sprachlicher und praktischer Interaktionsprozesse“ (Dausendschön-Gay, Domke \& Ohlhus 2010: 5).

Wissensgesellschaften sind wesentlich dadurch charakterisiert, dass sie diskursive Praktiken bzw. Gattungen (vgl. zu diesen und anderen grundlegenden Konzepten das Glossar in diesem Band) für die Erzeugung, Absicherung und Transmission von Wissen herausbilden. Im „kommunikativen Haushalt“ einer Gesellschaft (Luckmann 1986) lassen sich also Gattungen ausmachen, die in besonderer Weise auf die Kommunikation von Wissen spezialisiert sind (Deppermann 2015). So zeigt die Erfassung von Gattungsrepertoires in Unterrichtsgesprächen in den Fächern Deutsch und Mathematik, dass Gattungen des Erklärens und Argumentierens mit besonders hoher Frequenz genutzt werden (Heller et al. 2017; Morek 2016). Ihre herausgehobene Bedeutung für den Unterricht erklärt sich aus ihrer spezifischen Funktionalität für das Lehren und Lernen: Während das Erklären darauf zugeschnitten ist, Probleme des Wissenstransfers und der Wissensdemonstration zu lösen, ermöglicht das Argumentieren die Behandlung divergenter Geltungsansprüche (u.a. Morek, Heller \& Quasthoff 2017; Quasthoff, Heller \& Morek 2017). Es ist diese besondere Funktionalität für die Generierung, Absicherung und Transmission von Wissen, die den Status des Erklärens und Argumentierens als prototypische „bildungssprachliche Praktiken“ begründet (Heller \& Morek 2015; Morek \& Heller 2012). Sie spielen nicht nur im Unterricht, sondern folgerichtig auch in der Wissenschaftskommunikation eine zentrale Rolle (u. a. Latour \& Woolgar 1986; Knorr-Cetina 1984; Kuhn \& Reiser 2009; Trautmann 2004; Grundler \& Vogt 2006; Ehlich 2014; Redder 2016).

Erklären und Argumentieren sind komplexe diskursive Praktiken, weil sie von den Interaktanten erhebliche koordinative Leistungen verlangen, die im Falle des Erklärens insbesondere die wechselseitige Anzeige des (Nicht-)Verfügens von Wissen (Morek 2012), im Falle des Argumentierens die Signalisierung der (Nicht-)Akzeptanz von Geltungsansprüchen betreffen (Heller 2012). Aus diesem Grund weisen beide Praktiken eine komplexe sequenzielle Organisation auf, die die Etablierung gattungsspezifischer interaktiver Rollen und die Bearbeitung einer Reihe gesprächsstruktureller Aufgaben umfasst. $\mathrm{Zu}$ der Komplexität, die dem Erklären und Argumentieren somit im Hinblick auf ihre Kontextualisierung im laufenden Gespräch zukommt, gesellen sich weitere Anforderungen in Bezug auf die 
Vertextung von Erklärungen und Argumenten. Diese betreffen jenen Ausschnitt des Interaktionsprozesses, in dem ein Interaktant in der Rolle des Erklärers bzw. Proponenten/Opponenten ein redezugübergreifendes big package (Sacks 1995, 354-59; i.F. „Äußerungspaket“) bzw. einen multi-unit turn (Schegloff 1996: 60) hervorbringen muss. Aus fachlich-inhaltlicher Perspektive beinhaltet dies die Verknüpfung von Aussagen zu einem globalen Zusammenhang bzw. komplexeren Wissensgefüge. Diese Ebene der Vertextung bildet meines Erachtens die Schaltstelle, an der Wissenskonstruktion und diskursive Praktiken aufs Engste miteinander verbunden sind. Mit Ehlich (1984) verstehe ich „Vertextung“ im Unterschied zu „Text“ als medialitätsübergreifendes Konzept, weil Eigenschaften von Textualität auch mündlichen Äußerungspaketen zukommen: Letztere weisen miteinander verknüpfte Aussagen auf und sind somit intern strukturiert; ihre Struktur wird mittels lexikalischer, morpho-syntaktischer, prosodischer und körperlich-visueller Formen für die Rezipient/innen erkennbar gemacht bzw. markiert (zu den drei Dimensionen von Diskurskompetenz s. Quasthoff, Heller \& Morek in diesem Band). Diese textuellen Eigenschaften ermöglichen es, Zusammenhänge zwischen Sachverhalten herzustellen und Wissensbestände durch Veranschaulichung, Abstraktion und Verallgemeinerung zu transformieren.

In unserem Videokorpus von Deutsch- und Mathematikstunden (vgl. Abschnitt 3) lässt sich nun beobachten, dass es gerade für Erklärungen und Argumente unterschiedliche Arten gibt, auf die Aussagen zu einem Äußerungspaket zusammengeschnürt werden. Es scheint also unterschiedliche Vertextungsverfahren zu geben, mittels derer Sprecher/innen explanative oder argumentative Äußerungspakete hervorbringen. Mit dem Instrumentarium der Textlinguistik, die bislang vor allem textsortenspezifische Großformen unterscheidet, lässt sich diese Vielfalt möglicher Vertextungen nicht zufriedenstellend fassen. In der Gesprächslinguistik sind zwar einige Verfahren beschrieben worden, beispielsweise Veranschaulichungsverfahren in der medizinischen Experten-Laien-Kommunikation (vgl. Brünner \& Gülich 2002; zu anderen Kontexten vgl. Birkner \& Ehmer 2013); es existieren jedoch noch keine Studien, die für fachunterrichtliche LehrLern-Diskurse relevante Vertextungsverfahren beschreiben. Ebenso bildete die mögliche Gattungsgebundenheit von Vertextungsverfahren bislang einen blinden Fleck. Dementsprechend unternimmt der vorliegende Beitrag den Versuch, im schulischen Fachunterricht genutzte Vertextungsverfahren des Erklärens und Argumentierens systematisch zu beschreiben. Dies soll erstens ermöglichen, Formen und Funktionen sprachlich-diskursiver Praktiken in fachlichen Erkenntnisund Lernprozessen genauer $\mathrm{zu}$ fassen. Zweitens stellt die Beschreibung von Vertextungsverfahren auch eine Voraussetzung dafür dar, die Anforderungen, die diese im Rahmen des schulischen Fachunterrichts an Schülerinnen und Schüler stellen, sichtbar zu machen. 
Wenngleich der Beitrag dieses Vorhaben nur in ersten Ansätzen einzulösen vermag, bildet doch das InterPass-Korpus von 140 videographierten Unterrichtsstunden in den Fächern Mathematik und Deutsch (s. u.) eine empirische Grundlage, auf der sich eine gewisse Bandbreite an Vertextungsverfahren rekonstruieren lässt. Zunächst wird in Abschnitt 2 der zu untersuchende Gegenstand genauer konturiert und zu verwandten Konzepten der Gesprächs- und Textlinguistik ins Verhältnis gesetzt. Einem ethnomethodologischen Forschungsansatz folgend wird in Abschnitt 3 ein analytischer Rahmen für die linguistische Beschreibung von Vertextungsverfahren entwickelt, der auf der Rekonstruktion aus der Teilnehmerperspektive fußt und die interaktiven Bedingungen der Nutzung von Vertextungsverfahren konsequent berücksichtigt. Von den insgesamt 17 rekonstruierten Vertextungsverfahren werden in Abschnitt 4 jeweils zwei explanative und argumentative Verfahren exemplarisch beschrieben. Dabei wird in einem zweiten Analyseschritt gezeigt, dass sich die rekonstruierten gattungsspezifischen Vertextungsverfahren zwei übergreifenden Modi des Vertextens zuordnen lassen: Sie operieren entweder situierend oder abstrahierend. Beide Modi werden jeweils über spezifische sprachliche, prosodische und körperlich-visuelle Ausdrucksformen hergestellt und erfüllen im Rahmen der Wissenskonstruktion und -vermittlung distinkte Funktionen. Im Zuge ihrer Beschreibung werden sich weitere Forschungsfragen eröffnen, die in Abschnitt 5 skizziert werden.

\section{Vertextungsverfahren: gattungsspezifische Praktiken für die sprachlich-diskursive Darstellung komplexer Zusammenhänge}

Obwohl der vorliegende Beitrag mündliche Verfahren der Vertextung zum Gegenstand hat, sollen im Folgenden auch textlinguistische Arbeiten diskutiert werden, um den Begriff der Vertextungsverfahren von ähnlichen Konzepten wie ,Vertextungsmuster oder ,Themenentfaltungsmuster abzugrenzen. Während in der Textlinguistik zunächst der Blick auf die Ganzheit von Texten und Grundmuster der Vertextung gerichtet wurde, untersuchte die Gesprächslinguistik, wie Wissensbestände unter Bedingungen der Interaktivität und Flüchtigkeit in eine verstehbare sprachliche Form gebracht werden. Vereinfacht gesagt bildete also in der Textlinguistik zunächst das fertige Textprodukt den Ausgangspunkt einer eher top-down vorgehenden Erfassung hierarchisierter Musterhaftigkeiten, während der bottomup operierende Beschreibungsansatz der Gesprächslinguistik ,kleinere‘ Verfahren im Visier hatte, mit denen ,Text` im Gespräch sequenziell und interaktiv konstituiert wird. 


\subsection{Textlinguistische Ansätze}

Die Konzepte der globalen Text-, Strategie- und Vertextungsmuster (Heinemann 2000a, b), der Themenentfaltungsmuster (Brinker 1992) und der referentiellen Bewegung (Klein \& von Stutterheim 1992) zielen auf die Beschreibung von Strukturen oberhalb der Satzebene. Wiewohl sie in ihrer Konzeption nicht auf das Medium des Schriftlichen beschränkt sind, zeichnen sie sich doch insgesamt stärker durch die für die Textlinguistik größtenteils typische top-downBeschreibung aus.

Heinemann versteht Vertextungsmuster als Teilkomponenten von umfassenderen Strategien zur Textherstellung im Medium des Mündlichen und Schriftlichen. Indem sie von konkreten Textexemplaren abstrahiert und als textstrukturelle Muster mental repräsentiert werden, fungieren sie laut Heinemann als Strategien für die „Vertextung des Sprechanliegens“ (2000: 357). Sie ermöglichen also dem Sprecher oder der Schreiberin (und ebenso den Rezipient/innen) die „Sequenzierung von illokutiven und propositionalen Einheiten und deren Strukturierung zu Teiltexten/Teiltextthemeneinheiten, immer verknüpft mit Formulierungs- und Verbalisierungsprozessen“ (2000: 357). Dabei geht Heinemann davon aus, dass für alle Beteiligten eine Quaestio als immanenter Orientierungspunkt transparent und leitend ist.

Im Vergleich zu Vertextungsmustern, die primär von der Textfunktion her konzipiert sind, sind die Konzepte der Themenentfaltungsmuster (Brinker 1992) und der referentiellen Bewegung (Klein \& von Stutterheim 1992) vornehmlich semantisch fundiert. Themenentfaltungsmuster bezeichnen das „Gefüge von Beziehungen zwischen dem Thema (definiert als Kern des Gesprächsinhalts) und den in den einzelnen Gesprächsschritten ausgedrückten Teilinhalten (Teilthemen)“ (Brinker \& Hagemann 2001: 1256). Zu den Grundformen der Textentfaltung, die sich in einer Gesellschaft herausgebildet haben, zählt Brinker die Deskription, Narration, Explikation und Argumentation. Anstatt diese Muster nun aber auf rekonstruktivem Wege zu beschreiben, wird unterstellt, dass sich thematische Muster des Argumentierens und Erklärens weitgehend an dem logisch-formalen Argumentationsmodell von Toulmin (1958) bzw. dem deduktiv-nomologischen Erklärmodell von Hempel \& Oppenheimer (1948) orientieren. Erst in jüngster Zeit entstehen empirisch fundierte Untersuchungen $\mathrm{zu}$ Themenentfaltungsmustern (Behrens \& Gätje 2016).

Das Konzept der referentiellen Bewegung von Klein \& von Stutterheim (1992, 2008) geht von der Annahme aus, dass mit Texten Fragen oder Probleme gelöst werden; dementsprechend seien Prinzipien des textinternen Aufbaus durch eine Quaestio festgelegt. Der Sprecher bzw. die Schreiberin muss zunächst die Textquaestio erkennen und sodann auf Grundlage der Einschätzung 
des Partnerwissens und der kontextuellen Erwartungen Wissenselemente auswählen. Diese müssen schließlich für die verbale Äußerung linearisiert und perspektiviert werden. Über das Nachzeichnen der „referentiellen Bewegung“ lässt sich laut Stutterheim \& Klein die dem Text zugrundeliegende „Gesamtvorstellung“ als komplexe kognitive Struktur ermitteln. Rekonstruieren lassen sich Typen referentieller Bewegung nur ausgehend von der interpretativ zu erschließenden Quaestio; wie dies methodisch kontrolliert zu bewerkstelligen ist, ist jedoch noch ungeklärt.

Wenngleich sich die vorgestellten Konzepte hinsichtlich ihrer Schwerpunktsetzungen und analytischen Zugänge unterscheiden, so haben sie doch einen primär sprecherzentrierten Fokus gemeinsam: Sie gehen von Sprecher/innen bzw. Schreiber/innen aus, die ihre ,Anliegen' unter Einsatz mentaler Strategien darzustellen versuchen. Die grundlegende Interaktivität der Textkonstitution in Gesprächen bleibt dabei allerdings weitgehend unberücksichtigt. Gesprächsgegenstände bzw. Quaestiones werden von den Beteiligten interaktiv etabliert, und nicht selten kommt es im Gesprächsverlauf zu Quaestio-Verschiebungen. Die Interaktivität ist auch für die Produktion von explanativen bzw. argumentativen Äußerungspaketen durch einen (temporär etablierten) primären Sprecher konstitutiv: Dieser schneidet seine Erklärung bzw. Argumentation auf das fortlaufend im Gespräch angezeigte Interesse und Wissen des Gegenübers zu. Letzterer bestimmt also nicht nur mit, ob und zu welcher Art von Äußerungspaket es kommt, sondern nimmt auch durch Bekundung des (Nicht-)Verstehens, Zweifelns, Akzeptierens usw. online auf die Äußerungsproduktion Einfluss (Goodwin 1995). Aus dieser Sicht scheint es als wenig wahrscheinlich, dass Sprecher/innen in Gesprächen gleichsam ,ungestört mentale Pläne für Vertextung abrufen und ,abarbeiten'.

Eine weitere Einschränkung besteht darin, dass die Differenzierung in Grundformen der Vertextung bzw. Themenentfaltung auf der Ebene der Gattung bzw. Textsorte ansetzt. Schon für das Erzählen ließen sich aber Varianten des von Labov \& Waletzky (1967) beschriebenen Strukturierungsmusters ausmachen, das Elaborieren und Dramatisieren (Hausendorf \& Quasthoff 2005). Angesichts der Vielgestaltigkeit des Erklärens und Argumentierens (zum Erklären-Wie, -Was, -Warum vgl. Klein 2001; zum persuasiven und explorativen Argumentieren vgl. Ehlich 2014) ist anzunehmen, dass Interaktanten aus einer noch weitaus größeren Bandbreite explanativer und argumentativer Vertextungsmöglichkeiten schöpfen können. In Reaktion auf dieses Problem entwickelt Feilke (2012, 2014) das Konzept der „Textprozedur“. Aus seiner handlungstheoretischen Perspektive stellen Textprozeduren keine auf das Textganze bezogenen Musterhaftigkeiten dar, sondern bilden kommunikativ-sprachliche Handlungskomponenten eines zu schreibenden Textes. Es handelt sich somit um „Einheiten mittlerer Größe zwischen Satz und Text“ (Feilke 2014: 14). Als „sozial schematisierte Handlungszüge in Texten“ (Feilke 
2014: 15) umfassen Textprozeduren ein Handlungsschema (bspw. Positionieren, Begründen, Konzedieren) und entsprechende Prozedurenausdrücke. In jüngster Zeit wurden Textprozeduren auch im Medium der Mündlichkeit untersucht (Marx \& Steinhoff 2017). Noch offen ist allerdings, wie und an welchen sequenziellen Positionen eines Textes bzw. Äußerungspakets die jeweiligen Textprozeduren greifen und wie sie zu einer globalen Struktur verknüpft werden.

\subsection{Gesprächslinguistische Ansätze}

Die Gesprächsforschung verfährt bei der Untersuchung sog. textkonstituierender Verfahren strikt sequenzanalytisch und rekonstruiert, wie Gesprächsbeteiligte Zug um Zug Themen etablieren und im Verlauf des Sprechens sprachliche und textuelle Strukturen finden, um komplexe Sachverhalte und subjektive Phänomene (bspw. Emotionen) in eine verstehbare Form zu bringen (vgl. Schwitalla 2006; Deppermann 2007). Für solche Prozesse haben sich unterschiedliche Begriffe etabliert: Textkonstitutions- bzw. -herstellungsverfahren (Gülich \& Kotschi 1987, 1996) und Formulierungsverfahren (Schwitalla 2006; Deppermann 2007). Differenziert wird zwischen Verfahren, mit denen im Gespräch neue Inhalte dargestellt werden, und Verfahren, mit denen auf bereits Geäußertes Bezug genommen wird (z. B. Paraphrasen und Reformulierungen sowie Redebewertungen und -kommentierungen). Des Weiteren können sich die Verfahren auf Äußerungseinheiten lokaler oder globaler Reichweite beziehen. Der vorliegende Beitrag fokussiert vornehmlich auf globale Verfahren, mit denen komplexere Äußerungspakete vertextet werden, und bezeichnet diese dementsprechend als Vertextungsverfahren. Letztere lassen sich danach differenzieren, dass mit ihnen jeweils spezifische Kohärenzrelationen hergestellt werden können. Vertextungsverfahren sind folglich auf einer strukturell niedrigeren Ebene als Textsorten bzw. Gattungen anzusetzen: Sie stellen Ressourcen dar ,zur Realisierung von token der komplexeren Schemata der Sachverhaltsdarstellung“ (Deppermann 2007: 227). Die im vorangegangenen Abschnitt diskutierten Vertextungsmuster sind demnach auf einer abstrakteren Ebene anzusiedeln: In konkreten Gesprächen werden sie vermittels unterschiedlicher Vertextungsverfahren realisiert. So legt Ohlhus (2014) eine Reihe narrativer Verfahren zur Herstellung von zeitlicher Ordnung, zur Fokalisierung der Darstellung sowie zur Kommentierung der Erzählung frei, mit denen das abstrakte Erzählschema auf sehr unterschiedliche Weise realisiert werden kann.

Einschlägige Praktiken, mit denen in der wissensvermittelnden Kommunikation Äußerungspakete vertextet werden, haben Brünner \& Gülich (2002) als Verfahren der Veranschaulichung rekonstruiert. Dazu zählen Metaphern, Vergleiche, Szenarios, Beispiele, Beispielerzählungen und Konkretisierungen. Ihnen 
ist gemeinsam, dass abstrakte Wissensbestände vorstellbar gemacht werden. Die genannten globalen Verfahren werden im Rahmen der Experten-LaienKommunikation von den Experten verwendet und dienen der verständlichen Vermittlung abstrakter Wissensbestände an die Laien und der Rückbindung an die Lebenswelt des Gegenübers.

Im Unterschied zu Veranschaulichungsverfahren zielen die von Drescher (1992) beschriebenen Verfahren des Verallgemeinerns auf eine Ablösung vom Einzelfall. So ist die generalisierende Paraphrase ein rückbezügliches Verfahren, mit dem die inhaltliche Progression verlangsamt und eine Vorgängeräußerung unter Wegfall von zeitlichen Spezifizierungen und Relativierungen aufgenommen wird; dabei werden konkrete Bezeichnungen bzw. episodische Beschreibungen durch allgemeinere Begriffe bzw. einen generischen Satz (Drescher 1992: 88) ersetzt. Beim Verfahren des generalisierenden Vergleichens leitet das Lexem „wie“ den Übergang vom Partikulären ins Allgemeine ein, wobei die generalisierende Wiederaufnahme eines vorangegangenen Äußerungssegments durch sprachliche Mittel der Allgemeinheit (Hyperonyme, Indefinita, Frequenzadverbien, generisches Präsens) erfolgt. Beide Verfahren werden vornehmlich in erörternder oder begründender Funktion, also argumentativ genutzt.

Die von Drescher beschriebenen Verfahren sind abstrahierenden Formulierungen, wie sie für die Experten-Laien-Kommunikation (Deppermann 2011) oder auch die Kommunikation zwischen medizinischen Experten beschrieben wurden (Lymer et al. 2014), sehr ähnlich. Es handelt sich dabei um Verfahren, mit denen eine zentrale Aussage (bei der von Schlinkmann 2018 untersuchten Theaterkommunikation z. B. „die Stückaussage“) aus einer zuvor im Gespräch produzierten Beschreibung oder Erzählung entwickelt wird (Deppermann 2007). Lymer et al. (2014) beobachten über mehrere Sequenzen hinweg, dass in Gesprächen über Fehlerquellen im Rahmen der diagnostischen Radiologie sukzessive abstrahierende Instruktionen formuliert werden (Lymer et al. 2017). In semantischer Hinsicht zeichnen sich abstrahierende Formulierungen dadurch aus, dass einzelfallbezogene, situativ verankerte und episodisch strukturierte Bedeutungen in vom Einzelfall abgelöste Schlüsselwörter oder Kernaussagen transformiert werden. In der Regel haben sie entweder eine abschließende Wirkung oder etablieren für das weitere Gespräch relevante Zwischenergebnisse.

Mit einem systematischen Gattungsbezug rekonstruieren Quasthoff \& Hartmann (1982) vier Verfahren - in ihrer Terminologie: semantische Typen - des Erklärens von Alltagsbegriffen: das Nennen von Beispielen, das Nennen eines bedeutungsgleichen Ausdrucks, das Nennen wesentlicher Merkmale sowie das Nennen von übergeordnetem Begriff und unterscheidendem Merkmal (genus proximum und differentia specifica). In ihren Daten treten die Verfahren auch in Kombination auf. Mit einem stärkeren Fokus auf die Konzeptbildung differen- 
ziert Rehbein (1982) in Bezug auf Erklärverfahren mehrsprachiger Kinder zwischen satzförmigen, abstrahierenden Verfahren, bspw. dem Nennen einer Regel, und illustrierenden Verfahren wie dem Beispiel-Geben (und bei Wortbedeutungserklärungen in der Zweitsprache auch dem thematisch fokussierten Umschreiben oder dem Abdriften). Komor (2013) sieht vom Beispiel-Geben zum Umschreiben und zur satzförmigen konditionalen Form von Bedeutungserklärungen einen zunehmenden Abstraktionsgrad gegeben.

Neben Belegen für unterschiedliche Vertextungsverfahren finden sich in einschlägigen Arbeiten auch Hinweise auf distinkte übergreifende Modi (s. u.) der Vertextung. So zeigt Kern (2003), dass Spielerklärungen von Kindern entweder in demonstrierender oder explizierender Weise vollzogen werden. Während das $D e$ monstrieren durch eine starke Orientierung am Ablauf sowie gestisches Darstellen zentraler Spielhandlungen gekennzeichnet ist, wird beim Explizieren von konkreten Spielhandlungen abstrahiert und es werden Regeln in Form generischer Aussagen formuliert (Kern 2003; Stude 2005). In ähnlicher Weise differenzieren Schwitalla (1991) und Becker-Mrotzek (1989) mit Blick auf rekonstruktive Gattungen das Illustrieren durch wiederkehrende Handlungsfolgen vom Illustrieren durch ein singuläres Ereignis. Die erste Variante ist verallgemeinernd: Die Akteure werden durch soziale Kategorien eingeführt, auf Orts- und Zeitangaben wird verzichtet, und auch das Präsens bringt eine zeitliche Allgemeingültigkeit zum Ausdruck. Demgegenüber dient die zweite Variante dazu, einen Sachverhalt exemplarisch zu veranschaulichen, vor allem durch Einführung der Akteure mit definiter Deixis, lokale und temporale Situierung und Wahl des Präteritums oder Perfekts. Analog beobachtet König (2013) in narrativen Interviews, dass die Interviewten unterschiedliche Verfahren der Redewiedergabe nutzen. Während bei einer rekonstruierenden Redewiedergabe durch Verweise auf Ort, Zeit und den jeweiligen Sprecher eine Authentisierung der Rede erfolgt, zeichnet sich die generalisierende Redewiedergabe durch den Verzicht auf zeitliche und räumliche Situierung und eine allgemeine Referenzierung auf Sprecher/innen (mit dem Indefinitpronomen man) aus. Die zweite Variante der Redewiedergabe wird somit zu einem Mittel der verallgemeinernden Typisierung von Personen. Unterschiedliche Darstellungsmodi, in denen Verfahren der Rekonstruktion operieren - berichtende und inszenierende - findet auch Schlinkmann (2018) im Rahmen der Theaterkommunikation.

In der Zusammenschau deutet sich somit an, dass es Vertextungsverfahren gibt, die sich vornehmlich durch Situierung und eine episodenhafte, veranschaulichende Vertextung auszeichnen, während andere eher von situativen Aspekten abstrahieren und kondensierend verfahren. Bereits Brünner \& Gülich (2002) differenzieren in Bezug auf die Arzt-Patienten-Kommunikation zwei Gruppen von Verfahren, mit denen (krankheitsbezogenes) Wissen vermittelt 
wird: Reformulierungen, Paraphrasen, Explizierungen bzw. Erklärungen ${ }^{1}$ einerseits und Veranschaulichungen andererseits (2002: 24). Ehmer (2013) greift diese Beobachtung auf und fasst diese unterschiedlichen Typen in Anlehnung an Clark \& Gerrig (1990) als „Modi des Signalisierens“.2 Ebenso differenziert Schlinkmann (2018) als analytische Beschreibungsebenen zum einen Verfahren (des Rekonstruierens) und zum anderen Darstellungsmodi, in denen jeweils Gruppen von Verfahren operieren. Diese Unterscheidung wird hier aufgegriffen und systematisch auf Vertextungsweisen von Äußerungspaketen bezogen (Abschnitt 4.3); es zeigt sich doch in den vorliegenden Daten, dass im Unterricht nicht nur eine Vielzahl explanativer und argumentativer Vertextungsverfahren genutzt wird, sondern diese auch in zwei grundlegend unterschiedlichen Modi des Vertextens operieren, die auf kontextspezifische Anforderungen der Wissenskommunikation zugeschnitten sind. Im folgenden Abschnitt werden nun die Konzepte ,Vertextungsverfahren“ und ,Modus‘ weiter spezifiziert.

\subsection{Eingrenzung des Phänomens}

Ist eine Erklärung oder Argumentation interaktiv vorbereitet, d. h. von einem der Beteiligten relevant gesetzt und somit erwartbar gemacht worden, so ist derjenige Interaktant, dem nun die Rolle des Erklärenden oder Begründenden zugewiesen ist, gefordert, einen globalen Zusammenhang explanativer oder argumentativer Art herzustellen (Quasthoff, Heller \& Morek 2017). Letzteres wird i.F. als Kernaufgabe des Erklärens oder Argumentierens bezeichnet.

Für einen ersten Zugang zum Phänomen der globalen Vertextungsverfahren betrachten wir eine Sequenz aus dem Deutschunterricht einer fünften Gymnasialklasse. In der Unterrichtsstunde werden „Kriterien für einen guten Brief“ besprochen. Die Deutschlehrerin liest zunächst eines der Kriterien vor: „Schreibe vollständig, ausführlich und genau“ (Z. 52-54). Das Kriterium ist allgemeingültig formuliert: Angesprochen wird ein unspezifischer Adressat; weder kommt zum Ausdruck, wann das Kriterium gilt, noch wie es umzusetzen ist. Mit ihrer Frage „was ist denn DA:mit gemeint;“ (Z. 55) setzt die Deutschlehrerin das Erklären des Kriteriums relevant, wobei das Interrogativpronomen, die Modalpartikel denn sowie das akzentuierte und gedehnte „DA:mit“ die Erklärbedürftigkeit kontextualisieren. Das in der Folge zu vertextende Äußerungspaket ist im Transkript grau hinterlegt.

1 Diese Gruppe von Verfahren wird nicht unter eine Oberkategorie subsumiert.

2 Clark \& Gerrig (1990: 765) differenzieren zwischen drei „methods of performing communicative acts“: ,indicating“, „demonstrating“ und „describing“. 
Beispiel 1: 01-1-DU-02-41364431-Hauptteil_Brief (leh: Deutschlehrer, nik: Nikolas)

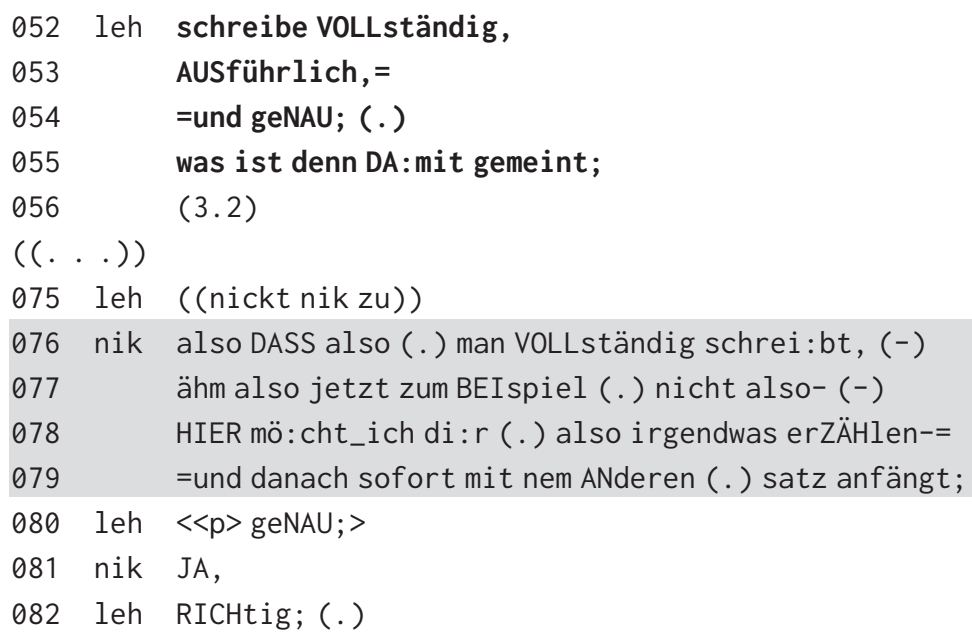

Auf den Zugzwang hin projiziert Nikolas mittels „also“ die Produktion eines Äußerungspakets (Z. 75-79). Dieses vertextet er mittels des Verfahrens Geben eines Beispiels. Dazu stellt er zunächst einen Bezug zum Explanandum her (Z. 75: „DASS also (.) man VOLLständig schrei:bt;“) und kündigt dann das Beispiel - hier: ein Negativbeispiel - mit Hilfe der Markierungsform „zum BEIspiel (.) nicht also“ an. Damit ist die Art der Hervorbringung des Äußerungspakets in Teilen vorgezeichnet: Es muss ein Beispiel genannt werden, das konkreter als die Kategorie ist, die es erklären soll (Brünner \& Gülich 2002: 34; Quasthoff \& Hartmann 1982: 105; Rettich 2011). ${ }^{3}$ Für „schreibe vollständig“ wird ein Beispiel in Form einer animierten Rede (Ehmer 2011) gegeben (Z. 78). Daran, dass hier nur ein Teil des Exemplificans (die Redeanimierung) einen maximalen Konkretheitsgrad aufweist, während das zweite Element eine weitere Texthandlung lediglich beschreibt (Z. 79: „und danach sofort mit nem ANderen (.) satz anfängt;“), wird deutlich, dass Beispiele nicht unbedingt maximal konkret, sondern lediglich konkreter als das Explanandum sein müssen. Insbesondere die Redeinszenierung erlaubt es Nikolas, den Rezipient/ innen anschaulich vorzuführen, was er unter mangelnder Vollständigkeit versteht.

3 Zu Beispielen in erklärender Funktion vgl. auch Rettich (2011), Morek (2012), Wyßuwa \& Beier (2013); zu Beispielen in argumentativer Funktion vgl. Müller \& Di Luzio (1995), Günthner (1995b), Rettich (2011), Heller (2012). Das Geben eines Beispiels ist somit eines der wenigen Vertextungsverfahren, das nicht auf eine Gattung spezialisiert ist (s. Kap. 4.3). 
Auf Grundlage dieser ersten Analyse lässt sich nun der Begriff des Vertextungsverfahrens weiter präzisieren. Vertextungsverfahren beziehen sich auf die Kernaufgabe, die sich dem Erklärer bzw. Proponenten/Opponenten (oder Erzähler) stellt. Vor dem Hintergrund eines Verständnisses von sprachlichem Handeln als sozialer Praxis sind Vertextungsverfahren als sozial etablierte und kulturell sedimentierte Praktiken zu verstehen, mit denen im Rahmen der gattungsspezifischen Kernaufgaben komplexe Sachverhalte unter Bedingungen der Interaktivität sukzessive in geordneter Weise dargestellt werden. Dabei können Verfahren auch miteinander kombiniert und wechselseitig ineinander eingebettet werden (Brünner \& Gülich 2002; Ehmer 2013; Linz 2018). Für Vertextungsverfahren haben sich bestimmte prosodische, körperlich-visuelle, lexikalische und morpho-syntaktische Formen sedimentiert, die jedoch nicht obligatorisch oder definitorisch für die einzelnen Verfahren sind (Deppermann 2007; Ohlhus 2014). Mit Blick auf das dreigliedrige Beschreibungsmodell von Diskurseinheiten (GLOBE, vgl. Hausendorf \& Quasthoff 2005 und Quasthoff, Heller \& Morek 2017, in diesem Band) gehören die Verfahren zur Ebene der pragmatisch-semantischen Mittel, $^{4}$ und zwar jener Mittel für die Durchführung des Kernjobs. ${ }^{5}$

Zur sukzessiven Herausbildung und Verfestigung von Vertextungsverfahren kommt es, wenn sich bestimmte kommunikativ zu bewerkstelligende Aufgaben oder Anforderungen in einem gesellschaftlichen Segment wiederkehrend stellen. Insofern stellen Verfahren - ebenso wie Gattungen im Großen (Günthner 1995) sowie Prozeduren (Feilke 2014), syntaktische Konstruktionen (Günthner 2006) und vorgeformte Ausdrücke (Dausendschön-Gay, Gülich \& Krafft 2007) im Kleinen - strukturelle Lösungen für wiederkehrende Probleme dar. Kompetente Sprecher/innen müssen also die Produktion komplexerer Äußerungspakete nicht immer wieder von Neuem planen, sondern können auf gesellschaftlich etablierte und damit mehr oder weniger vorgeprägte Verfahren der Vertextung zurückgreifen. In diesem Sinne stellen Vertextungsverfahren kontextfreie Ressourcen dar. Ihnen kommt deshalb eine Entlastung sowohl für die Produzenten als auch für die Rezipienten zu (Luckmann 2002). Gleichwohl stellen Vertextungsverfahren keine ,Fertigteile‘ dar, sondern eher Modelle, an denen sich Interaktanten orien-

\footnotetext{
4 GLOBE umfasst drei Beschreibungsebenen: Die interaktiv zu bearbeitenden konversationellen Aufgaben bzw. Jobs des Erzählens, Erklärens, Argumentierens werden von jedem der Gesprächsbeteiligten Zug um Zug mit Hilfe von pragmatisch-semantischen Mitteln bewerkstelligt. Die Mittel wiederum werden durch sprachliche, prosodische und nonverbale Formen realisiert.

5 Wollte man Vertextungsverfahren in die drei Beschreibungsebenen kommunikativer Gattungen (Günthner 1995) einordnen, so wären sie auf der Binnenebene anzusiedeln.
} 
tieren können (Dausendschön-Gay, Gülich \& Krafft 2007: 184f.). Sie müssen kontextsensitiv an den jeweiligen Gesprächskontext angebunden und auf die jeweiligen Rezipient/innen zugeschnitten werden. Der jeweilige Gesprächskontext kann darüber hinaus einen bestimmten Darstellungsmodus nahelegen, der entweder situierend oder abstrahierend sein kann. Vertextungsverfahren lassen sich also hinsichtlich bestimmter Modi differenzieren, die im Rahmen der Wissenskonstruktion und -vermittlung distinkte epistemische Funktionen erfüllen. Im obigen Fall wird mit dem globalen Zugzwang der Lehrerin die Erwartung etabliert, die Bedeutung eines abstrakt formulierten Kriteriums verständlich zu machen. Genau dies leistet das von Nikolas gewählte Geben eines Beispiels: Es operiert in einem situierenden Modus, der darauf ausgerichtet ist, Abstraktes („schreibe vollständig“) durch Veranschaulichen oder gar Vorführen konkreter Bezugshandlungen zu verdeutlichen.

\section{Daten und analytisches Vorgehen}

Auch wenn die Ausformung und Verfestigung von Verfahren in einer Sprachgemeinschaft letztlich aus wiederkehrenden kommunikativen Aufgaben resultiert, wird hier ein methodischer Weg eingeschlagen, der Verfahren nicht ausgehend von den immanenten Anforderungen des jeweiligen Erklär- oder Argumentationsgegenstands beschreibt. Dies ist schon deshalb nicht zielführend, weil letztere gerade mittels unterschiedlicher Verfahren bearbeitet werden können. Vielmehr wird rekonstruiert, was aus Perspektive der Beteiligten als (funktionales) Vertextungsverfahren gilt. Die Analyse muss dabei allerdings dem Umstand Rechnung tragen, dass sie auf Interaktionsdaten basiert, an denen - im Hinblick auf das Erklären und Argumentieren - noch nicht vollends kompetente Sprecher/innen beteiligt sind. Die Rekonstruktion steht somit vor dem Problem, ausgebaute Exemplare von Vertextungsverfahren von nur rudimentär realisierten unterscheiden zu müssen. Dieses Problem wird auf zweierlei Weise gelöst: Erstens fokussiert die Analyse auf diskursiv relativ kompetente Sprecher/innen. Dazu zählen neben den Lehrpersonen auch Schülerinnen und Schüler, deren diskursstrukturelle Profile (vgl. Heller 2017b) auf eine vergleichsweise hohe Diskurskompetenz schließen lassen. Zweitens werden die im Medium des Mündlichen beobachtbaren Verfahren der Verständigungssicherung und Verstehensdokumentation (Deppermann \& Schmitt 2008) sowie der Redebewertung und -kommentierung (Gülich \& Kotschi 1987) systematisch berücksichtigt: Sie werden in den untersuchten Unterrichtsgesprächen vordringlich von der Lehrperson realisiert und geben weiteren Aufschluss über die Erwartung kompetenter 
Sprecher/innen an die ,Normalform“ eines Vertextungsverfahrens. In diesem Sinne werden auch Vorkommen kollaborativer Vertextungen in die Analyse einbezogen.

Die Analyseeinheit bilden explanative und argumentative Diskurseinheiten. Es werden also nicht nur die Kernjobs - die Hervorbringung des explanativen oder argumentativen Äußerungspakets -, sondern auch deren interaktive Vorund Nachbereitung (s. Abb. 1) systematisch in die Analyse einbezogen:

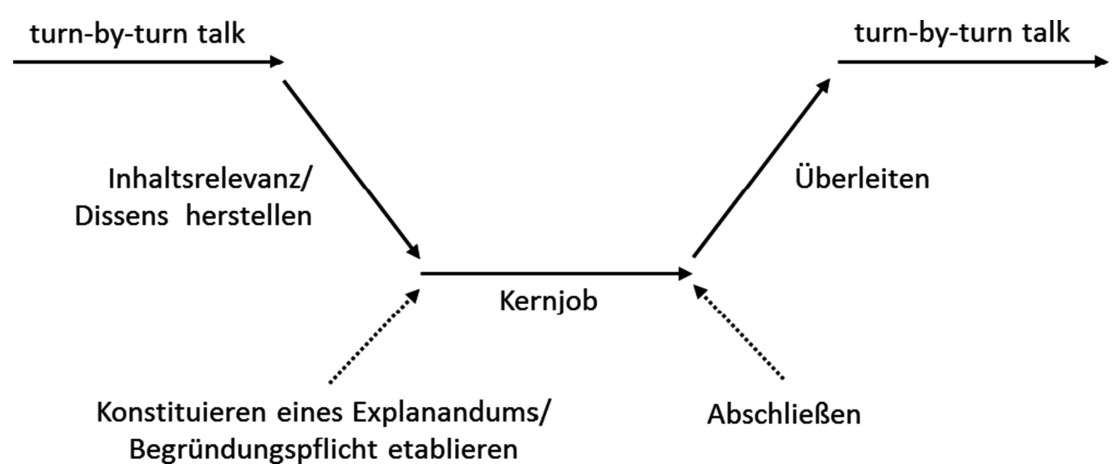

Abb. 1: Gesprächsaufgaben beim Erklären und Argumentieren (Quasthoff, Heller \& Morek 2017).

Auf dieser Grundlage wird zunächst rekonstruiert, an welcher kommunikativen Gattung sich die Beteiligten orientieren. Für die Analyse der Vertextungsverfahren wird sodann auf die Kernaufgabe des Vertextens fokussiert. Dabei wird die Herstellung globalsemantischer Zusammenhänge unter der Berücksichtigung der Interaktivität so nachgezeichnet, wie sie im jeweiligen Äußerungspaket sukzessive durch Hinweise auf inhaltliche Relationen nahegelegt wird. Da diese Hinweise nicht nur mittels verbalsprachlicher, sondern auch prosodischer und körperlich-visueller Ressourcen erfolgen, werden diese systematisch in die Analyse einbezogen, um die Bündel bzw. Konfigurationen semiotischer Ressourcen (Goodwin 2013) herauszupräparieren, die die jeweilige Erklärung bzw. Argumentation hervorbringen und markieren.

Es wird nur dann von einem Vertextungsverfahren gesprochen, wenn sich in den Daten mehrere Aufweise des entsprechenden Vorgehens finden. Diese sind vergleichend untersucht worden, um zentrale Konstituenten des Verfahrens $\mathrm{zu}$ bestimmen. In einem weiteren Schritt wurden die auf diese Weise rekonstruierten 17 Verfahren im Hinblick auf Modi des Vertextens untersucht und zwei Gruppen, situierenden und abstrahierenden Verfahren, zugeordnet. 
Die Datengrundlage bilden Unterrichtsgespräche in den Fächern Deutsch und Mathematik, die im Rahmen des BMBF-Projektes InterPass ${ }^{6}$ in fünf fünften Klassen an Gesamtschulen und Gymnasien videographisch aufgezeichnet wurden. Die Aufzeichnung erfolgte mit vier Kameras, von denen eine auf die Lehrperson gerichtet war, eine die Totale erfasste und zwei weitere jeweils einen Gruppentisch fokussierten. Die insgesamt 140 Unterrichtsstunden wurden im Transkriptionseditor Transana hinsichtlich der Sozialformen, Themen und Gattungen annotiert. Von den Unterrichtsgesprächen, die ca. 69\% der gesamten Unterrichtszeit ausmachten, wurden 358 Sequenzen rekonstruktiver, deskriptiver, explanativer und argumentativer Art transkribiert (s. die Transkriptionskonventionen im Anhang des Bandes). Die Untersuchung von Vertextungsverfahren fokussiert auf explanative und argumentative Sequenzen, da diese in beiden Fächern mit Abstand am häufigsten anzutreffen waren.

\section{Vertextungsverfahren des Erklärens und Argumentierens: exemplarische Analysen}

\subsection{Vertextungsverfahren des Erklärens}

Im Folgenden werden zwei Vertextungsverfahren des Erklärens, das schrittweise Vorführen eines Vorgehens und das Formulieren einer Kernaussage, an jeweils einer Sequenz exemplarisch untersucht. Der Analyse vorgreifend sei zur besseren Übersicht vorausgeschickt, dass die beiden Verfahren zugleich zwei Modi des Vertextens abbilden. Während das schrittweise Vorführen eines Vorgehens einem situierenden Modus folgt, stellt das Formulieren einer Kernaussage ein abstrahierendes Verfahren dar.

\subsubsection{Schrittweises Vorführen eines Vorgehens}

Das schrittweise Vorführen eines Vorgehens ist ein Verfahren, mit dem komplexere Operationen körperlicher, mechanischer, kognitiver o. ä. Art gleichsam dekomprimiert oder entpackt werden. In Beispiel 2 haben wir es mit der im Mathematikunter-

6 Das Projekt InterPass wurde mit Mitteln des Bundesministeriums für Bildung und Forschung unter dem Förderkennzeichen 01JC1112 in der Förderlinie „Teilhabe und Chancengerechtigkeit“ gefördert. Leitung: Susanne Prediger (TU Dortmund, Mathematikdidaktik) und Uta Quasthoff (TU Dortmund, Sprachwissenschaft und Sprachdidaktik). 
richt wiederkehrenden Situation $\mathrm{zu}$ tun, dass unterschiedliche Lösungen $\mathrm{zu}$ einer Aufgabe existieren. Die Sachaufgabe „Wie viel wiegt ein Blauwal, der morgens 120 Tonnen wiegt, nachdem er 4 Millionen Krill gefressen hat?“ beinhaltet das Umrechnen von Einheiten (Gramm, Kilogramm, Tonnen). Zwei Lösungen stehen bereits an der Tafel, als Neala aufgefordert wird, ihre Lösung ebenfalls anzuschreiben.

Beispiel 2. 02-5-MU-06-00170110-Nealas Lösung Tafel:

$\begin{array}{|ll|}\text { Neala } & \\ 2 \mathrm{~g} \cdot 4 \text { mio } & =8 \text { mio g } \\ 8 \text { miog } & =800 \mathrm{~kg} \\ 120000 \mathrm{~kg}+800 \mathrm{~kg} & =120800 \mathrm{~kg} \\ 120800 \mathrm{~kg} & =121 \dagger\end{array}$

001 leh gAnz viele haben jetzt grad (.) schon gesagt sie verSTEHen nicht was du gemacht hast; $(-)$

002 [von daher $(-)$ versuch einfach-]

003 sus [murmeln ]

004 leh ((blickt in die Klasse, schüttelt Kopf))

005 nea ALso.

006 leh verSUCH einfach mal zu erklären <p>> was du gemacht hast; >

007 nea ich hab die |ZWEI gramm| von dem krill genommen, (--) $\mid(($ zeigt $)) \mid$

008 und dann hab ich äh die | vier milLIOnen | genommen, = I((zeigt)) |

$009=$ =weil (-) äh der blauwal frisst ja (-) vier millionen mal die zwei GRAMM. (---)

010 das sind dann |!ACHT! millionen gramm. | (-)

$$
\text { I ((zeigt)) }
$$

011 und die acht millionen GRAMM, (.)

012 sind gleich achthundert KIlogramm. (---)

013 und dann hab ich die (.) hundert (.)zwanzig TONnen genommen, =

$014=$ =gleich hundertzwanzigtausend kilogramm, (-)

015 plus die ACHThundert kilogramm, =

$016 \quad$ das sind hundert (.) zwanzigtausendACHThundert kilogramm, (--) 


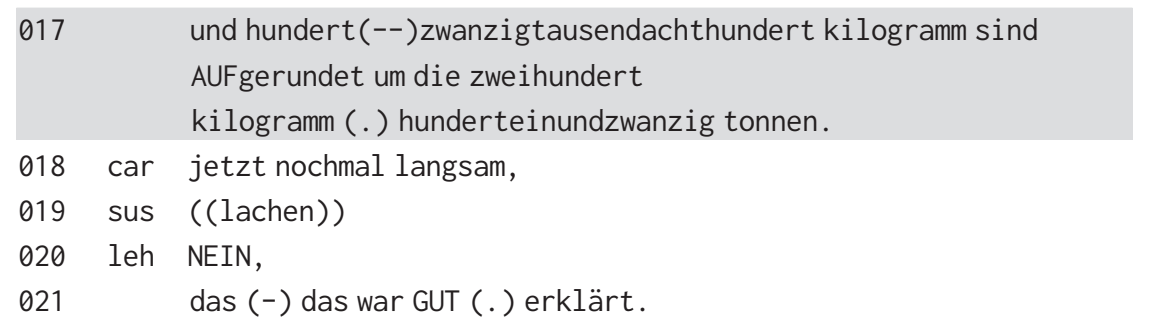

Der lehrerseitige Zugzwang wird eingeleitet, indem der Lehrer die während des Schreibens zu hörenden Schülerkommentare zusammenfassend wiedergibt. Die Redewiedergabe „sie verSTEHen nicht was du gemacht hast;“ (Z. 1) leistet zweierlei: Zum einen etabliert sie ein Explanandum, das das Wie des mathematischen Vorgehens betrifft. Zum anderen zeigt sie Neala als der prospektiven Erklärerin an, dass ihre Erklärung vor allem auf Nachvollziehbarkeit für die Mitlernenden zielen soll, die somit als primäre Adressaten der Erklärung etabliert werden. Wir werden an späterer Stelle noch darauf zurückkommen, dass der Zugzwang, der in Z. 6 auch noch einmal expliziert wird, damit klar in einen situierenden Modus des Vertextens steuert.

Betrachten wir nun Nealas Äußerungspaket: Mit ,ich hab die ZWEI gramm von dem krill genommen,“ (Z. 7) versetzt Neala die Rezipient/innen zeitlich an den Anfangspunkt ihrer Operation zurück und führt sie dann chronologisch durch die lineare Abfolge mathematischer Schritte (Additionen, Umwandlungen von Einheiten, Aufrunden). Diese perspektiviert sie mit dem Verb nehmen als praktische, manuell ausführbare Handlungen (Z. 7, 8, 13). Die Teiloperationen werden zumeist einfach genannt (Z. 7-8, 11-12, 13-14, 15); in Einzelfällen werden sie auch durch Angabe von Gründen genauer plausibilisiert (Z. 9: , = weil (-) äh der blauwal frisst ja (-) vier millionen mal die zwei GRAMM.“). Im Großen und Ganzen orientiert sich also die Struktur der Vertextung an der zeitlichen Abfolge der Teiloperationen.

Durch die Konfigurierung jeweils unterschiedlicher semiotischer Ressourcen markiert Neala Teilschritte und Zwischenresultate systematisch unterschiedlich. Für die Nennung der Schritte wählt sie durchgängig das Perfekt; am Ende eines jeden Schrittes produziert sie eine mittelsteigende Tonhöhenbewegung, die den Rezipient/innen anzeigt, dass als nächstes entweder ein weiterer Schritt oder ein Zwischenergebnis zu erwarten ist. Die Verkettung von Schritten erfolgt stets mit „und dann“. Demgegenüber werden (Zwischen-)Resultate (Z. 10, 12, 17, 18) durchgängig mit dem Kopulaverb sein im Präsens markiert und mit einer stark fallenden Tonhöhenbewegung abgeschlossen. Die systematisch unterschiedlichen Markierungen bewirken, dass die Reihe von Schritten, in die die komplexe Operation hier zerlegt wird, erkennbar in Abschnitte segmentiert wird. Die Segmentierung wird durch längere Pausen am Turnende (Z. 9, 12, 17) zusätzlich kenntlich gemacht. Den Rezipient/innen wird auf diese Weise Zeit gegeben, die einzelnen Operationen 
und deren Resultate nachzuvollziehen bzw. „sacken zu lassen“ (Keppler 1989). Das Verfahren zeichnet sich weiterhin dadurch aus, dass die Chronologie der Schritte durch das Zeigen auf Elemente der Gleichung an der Tafel veranschaulicht wird. Die Rezipient/innen werden dadurch zu Zuschauer/innen gemacht und auch visuell Schritt für Schritt geführt.

Beim Vorführen eines Vorgehens wird also eine komplexe Operation in kleinere Schritte zergliedert. Diese Dekomprimierung geht mit einer linear-sequenzierenden Vertextung (Quasthoff 2012) einher. Um die schrittweise Ausführung einer komplexen Operation beobachtbar zu machen, werden mathematische Operationen nicht nur als praktische Handlungen perspektiviert, sondern durch Zeigehandlungen auf den Text (und in anderen Fällen auf Objekte oder mittels darstellender Gesten, vgl. Heller 2016; Kääntä, Kasper \& Piirainen-Marsh 2018) konkretisiert. Die Leistung dieser zeitlichen Koordinierung verbaler und gestischer Äußerungsteile besteht in einer stärkeren Situierung und Nachvollziehbarkeit. Zudem werden einzelne Schritte getrennt voneinander bearbeitbar und Fehler auf diese Weise korrigierbar (Harren 2015: 157).

Das schrittweise Vorführen eines Vorgehens findet sich in unserem Korpus ausschließlich beim Erklären-Wie. In einer Variante wird es allerdings auch im Rahmen argumentativer Sequenzen verwendet, wenn die fachliche Richtigkeit oder Funktionalität eines Lösungsansatzes durch Vorführen des Vorgehens demonstriert wird. Allerdings ist das Vorführen dann stets mit einer These oder Folgerung kombiniert, in der die Richtigkeit des Vorgehens behauptet oder abgeleitet wird. Dies erhöht die strukturelle Komplexität des Verfahrens, die sich nicht mehr durchgängig an der Chronologie der Schritte orientieren kann.

\subsubsection{Verdichten zu einer Kernaussage}

Das Verdichten zu einer Kernaussage ist ein Verfahren, das den abstrahierenden Formulierungen (s. Abschnitt 2.2) ähnelt. Es dient dazu, zentrale Aussagen von Texten, szenischen Darstellungen (vgl. Schlinkmann 2018 im Rahmen der Theaterkommunikation) oder auch beobachteten Handlungen zu entwickeln. In Beispiel 2 sind die Schülerinnen und Schüler gefordert, die „Hauptproblematik“ eines gerade in der Schule gesehenen Theaterstückes herauszuarbeiten, in dem es um die Freundschaft zwischen Billy und dem Außenseiter Gerald, einem Gorilla, geht. Nachdem die Fünftklässlerin Darja auf die erste Frage des Lehrers nach dem „Problem“ des Stückes zunächst auf einzelne Szenen Bezug genommen hatte, kommentiert der Lehrer Darjas Beitrag metakommunikativ und reformuliert dann den Zugzwang (Z. 38). Im Anschluss daran sehen wir zwei Lernende, Kevin und Mia, beim Verdichten zu einer Kernaussage. 
Beispiel 3. 02-2-DU-03-11021320-Kernproblematik

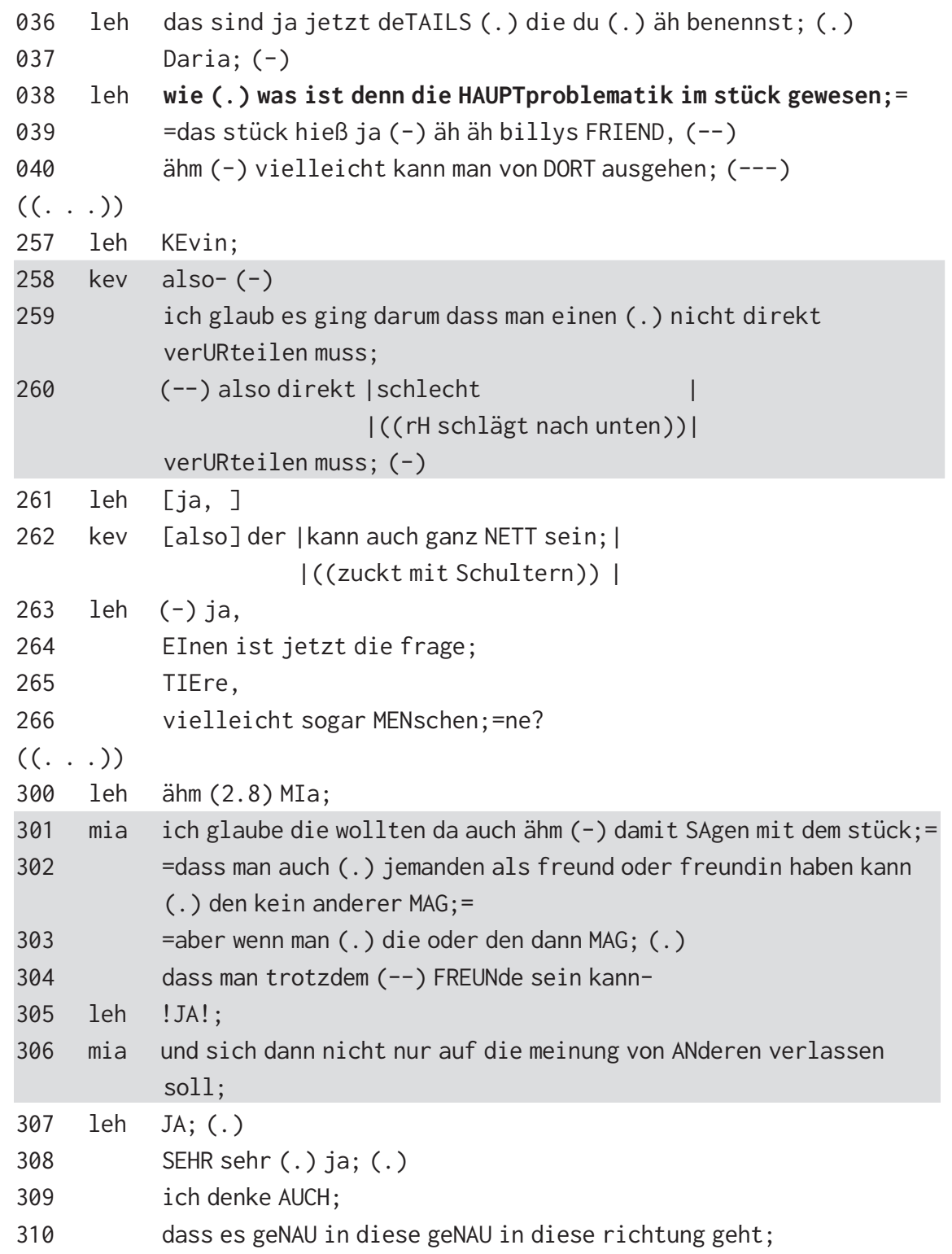


Die Frage des Lehrers baut einen Kontrast zu dem in der Redekommentierung Geäußerten (Z. 36: „das sind ja jetzt deTAILS“) auf: Der Fokusakzent auf „HAUPT“ zeigt an, dass es nicht um Detailwissen, sondern um das Herausarbeiten eines die Geschichte im Kern ausmachenden Problems geht. Dass dieses Problem nur unterhalb der Oberfläche der Handlung zu ,finden“ und ,herauszuarbeiten' ist, wird in dem Hinweis „das stück hieß ja (-) äh äh billys FRIEND, (-) ähm (-) vielleicht kann man von DORT ausgehen“ deutlich, der den Rezipient/innen eine Art Anleitung für die Erfüllung des Zugzwangs gibt.

Nach mehreren Versuchen anderer Schülerinnen und Schüler erhält Kevin das Rederecht. Nachdem er mit dem Diskursmarker „also“ sein Äußerungspaket projiziert und das Folgende mit dem epistemic stance marker (Kärkkaiinen 2006) „ich glaub“ als tentativ markiert, formuliert er eine allgemeine Moral des Stückes (Z. 259-260). Betrachten wir die Konfiguration semiotischer Ressourcen, so fällt auf, dass das Korrelat es zusammen mit dem Verbgefüge darum gehen auf das Theaterstück als Ganzes referenziert. Die vorgeformte Wendung „es geht darum, dass“ zwingt den Sprecher also quasi dazu, etwas für das gesamte Stück Wesentliches zu formulieren. Dies löst Kevin nun im dass-Komplement ein: Sich vom im Theaterstück dargestellten Einzelfall lösend, arbeitet er mit „dass man einen (.) nicht direkt verURteilen muss;“ eine allgemeine Moral bzw. Lehre (Ayaß 2013) heraus. Die Handlung des Theaterstückes mitsamt ihrer lokalen, zeitlichen und personellen Situierung wird ausgeblendet; stattdessen wird sie auf ihre Implikation für die mit dem Indefinitpronomen „man“ referenzierte Allgemeinheit kondensiert. Indem Kevin zusätzlich das unbestimmte „einen“ als Pronomen wählt, verleiht er der Aussage eine verallgemeinernde Qualität und macht sie auf andere Fälle übertragbar. Dass für das Verdichten einer solchen Moral Genauigkeit eine zentrale Rolle spielt, wird an Kevins Reformulierung (Z. 260) sichtbar, mit der er seine Aussage zu spezifizieren versucht. Dem eingefügten „schlecht“ wird dabei mit einem beat (McNeill 1992) zusätzlich Prägnanz verliehen. Es ist anzunehmen, dass aus Kevins Sicht der Zugzwang damit erfüllt ist. Zwar erläutert er die formulierte Kernaussage noch mit der Ergänzung „der kann ja auch ganz NETT sein“, doch geschieht dies erst nach einer Pause und auf einen continuer des Lehrers (Z. 261) hin.

In seiner Reaktion „EInen ist jetzt die frage;“ macht der Lehrer zum einen deutlich, dass ein Stand erreicht ist, von dem aus weitergearbeitet werden kann. Zugleich steuert er die Richtung der Weiterarbeit, indem er ein Ausfüllen des bislang inhaltsleeren Platzhalters „EInen“ einfordert. Mit „TIEre“ und „MENschen“ bringt er als Füller allgemeine Kategorien ins Spiel, auf die sich die von Kevin formulierte Moral beziehen lässt. Der lehrerseitige Umgang mit Kevins Formulierung einer Kernaussage zeigt den Gesprächsbeteiligten ebenso wie uns somit an, dass Kevins 
Formulierung noch etwas fehlt: Es müssen Kategorien gefunden werden, die angeben, auf welche Klasse von Fällen die Lehre anwendbar ist. Wir haben hier also aus Sicht des Lehrers ein noch nicht gänzlich geglücktes Verdichten zu einer Kernaussage vor uns. Demgegenüber erfährt der Versuch von Mia eine emphatische Bestätigung (Z. 308-309). Was unterscheidet ihre Vertextung von derjenigen Kevins?

Auch Mias Kernaussage enthält zwei Teile, eine redeeinleitende Formel (die wie bei Kevin mit einem epistemic stance marker eingeleitet wird) und die eigentliche Aussage. Die redeeinleitende Formel weicht von den für Redeinszenierungen üblichen Quotativen in mehrerlei Hinsicht ab: Das deiktische „die“ referenziert in unpersönlicher Weise auf die ,Macher‘ (den Autor, die Darsteller) des Theaterstücks; das Modalverb wollen zusammen mit dem Verbgefüge damit sagen verweist auf deren mit dem Stück verbundene Intention. Mit der einleitenden Formel wird also keine Rede angekündigt, sondern eine den Machern zwar zuzuschreibende, aber von der Sprecherin selbst herauszuarbeitende Aussage (vgl. im Rahmen der Theater- bzw. Kunstkommunikation: Schlinkmann 2018; Hausendorf 2011). Für die Formulierung der Aussage etabliert Mia nun anders als Kevin eine zwar allgemeine, doch ausreichend spezifische Kategorie: „Freund, den kein anderer mag“. Mit solchen konversationellen Kategorisierungen (Oberzaucher \& DausendschönGay 2014: 373) werden interaktiv ,inferenzielle Schlüsse [erarbeitet], die nicht als Komponenten des kulturellen Gedächtnisses abgerufen werden können, sondern die erst für den aktuellen Bedarf der Aufgabenstellung hergestellt werden müssen. “ Im vorliegenden Fall werden sie zur Sinnexplikation und Sinndeutung eingesetzt. Auch in Mias Beitrag wird eine Orientierung an Genauigkeit in ihrer Formulierungsarbeit sichtbar: Die mit dem Indefinitpronomen „man“ generisch formulierte Kernaussage wird noch einmal reformuliert (Z. 303-304) und durch ein weiteres mit der Moral verbundenes Handlungsprinzip ergänzt (Z. 306: „und sich dann nicht nur auf die meinung von anderen verlassen soll;“). Bei alledem wird der Allgemeinheitsgrad beibehalten, indem durchgängig Indefinitpronomen (man, jemandem, anderen) verwendet werden. Durch die Referenzierung auf nicht näher identifizierbare Personen wird die Aussage vom konkreten Fall des Theaterstücks abgelöst und auf andere Fälle übertragbar gemacht. Diese Transportabilität der Aussage wird außerdem durch die einprägsame satzförmige Form erhöht.

Das Verdichten zu einer Kernaussage ist also ein auf Verknappung ausgelegtes Vertextungsverfahren. In unseren Daten wird es im Kontext des Erklären-Was ${ }^{7}$

7 Das Erklären-Was (Klein 2001) kann sich diesem Verständnis nach nicht nur auf das Erklären von Wortbedeutungen, sondern auch auf die Bedeutung größerer Einheiten wie Theaterstücke, Texte usw. beziehen. 
genutzt. Die Anforderung beim Verdichten zu Kernaussagen besteht nicht wie beim schrittweisen Vorführen eines Vorgehens darin, eine komplexe Operation in eine geordnete Kette von Teilhandlungen zu zerlegen. Vielmehr ist gerade das Gegenteil gefordert: Aus einer kontextuell verankerten, episodisch detailreich dargestellten ,Vorlage“ wird das Wesentliche abstrahiert. Das ,Material', das verdichtet wird, bilden jeweils komplexere mündliche oder schriftliche Texte oder beobachtete Handlungen. Die Kernaussage kann dabei die Form einer Moral, Maxime, Intention, aber auch eines Ziels (das bspw. aus einer mathematischen Sachaufgabe entnommen werden soll) annehmen. Ihre Versprachlichung besteht aus (1) einer generischen redeeinleitenden Formel und (2) der eigentlichen Kernaussage. Vorgeformte Ausdrücke wie es geht darum, dass oder die wollen damit sagen, dass bieten gleichsam einen Steigbügel in das kondensierende Formulieren (Czicza 2015), das stets eine Abstraktion beinhaltet und durch Etablieren allgemeiner und zugleich ausreichend spezifischer Kategorien eine Übertragbarkeit auf andere Fälle ermöglicht.

Ein verwandtes Verfahren ist das Formulieren einer Anwendungsbedingung oder Konvention (vgl. Kap. 4.3). Dieses wird jedoch im Rahmen des Erklärens von Vorgehensweisen (Erklären-Wie) oder auch des Argumentierens verwendet. Dabei wird aus einem bereits praktizierten oder beobachteten Vorgehen eine Anwendungsbedingung oder Konvention herausgearbeitet; einleitende Wendungen (es geht darum, dass) entfallen; die Regel wird zumeist im Rahmen einer konditionalen Konstruktion formuliert, wobei die Regelhaftigkeit durch Frequenzadverbien (immer) markiert wird.

\subsection{Vertextungsverfahren des Argumentierens}

Analog zum vorigen Abschnitt werden im Folgenden zwei der rekonstruierten argumentativen Vertextungsverfahren exemplarisch vorgestellt, das explizierende Paraphrasieren von Belegen für eine Deutung und das Etablieren und Nutzen einer Schlussregel. Während dem erstgenannten Verfahren ein situierender Modus zugrunde liegt, verfährt das zweite abstrahierend.

\subsubsection{Explizierendes Paraphrasieren von Belegen für eine Deutung}

Das explizierende Paraphrasieren von Belegen für eine Deutung bildet gleichsam das Gegenstück zu der von Drescher (1992) beschriebenen generalisierenden Paraphrase. Es wird in Kontexten genutzt, in denen sich die Beteiligten über ihre Deutungen von Texten, Bildern, Filmen usw. verständigen. Als Träger des kulturellen Gedächtnisses sind Texte (ebenso wie andere kulturelle Erzeugnisse) Raible zufolge grundsätzlich 
auslegungsbedürftig: Sie weisen Leerstellen auf, die vom Leser gefüllt werden müssen. Darüber hinaus können sie eine Diskurswelt evozieren oder eine Sprache nutzen, die dem Leser nicht vertraut ist. Texte bedürfen also der „Kommentierung“ (Raible 1995) bzw. der Deutung als einer Grundform des Verstehens. Hausendorf (2016: 499) fasst Deuten als ein Verfahren für die Beantwortung der Frage ,Was steckt dahinter?‘. Deutungen werden also dann relevant, wenn die Kommunikation die Domäne des Wahrnehmbaren verlässt und etwas Wahrgenommenes verstehbar gemacht werden soll. Dementsprechend sind Deutungen häufig tentativ und zeichnen sich durch epistemische Modalisierungen aus. Damit einher geht, dass die Redewiedergaben, Paraphrasierungen und Explizierungen (Schlinkmann 2018), mittels derer Deutungen vollzogen werden, vor allem in argumentativen Kontexten auftreten. In unseren Daten lässt sich dieses Vertextungsverfahren primär im Deutschunterricht beobachten. Wenngleich unser Korpus keine weitergehenden Aussagen erlaubt, ist doch anzunehmen, dass dies auch für andere textwissenschaftlich orientierte Fächer gilt (s. bspw. zur Interpretation historischer Quellen Handro 2013).

In Beispiel 4 wird eine Geschichte im Hinblick auf ihren formalen Aufbau (Einleitung, Hauptteil, Schluss) untersucht. Es liegen unterschiedliche Auffassungen zu der Frage vor, welche Textteile zur Einleitung gehören. Mit der Entscheidungsfrage „Wer meint, dass die Einleitung nicht in Zeile neun endet?“ hat die Lehrerin von der Klasse eine Stellungnahme mittels Melden eingefordert, so dass der Dissens nun für alle sichtbar hergestellt ist. Mit dem nächsten Zugzwang etabliert die Lehrerin eine Begründungspflicht (Heller 2012): Es sollen Argumente für eine Lesart der Textstelle vorgebracht werden, die diese der Einleitung zuschlägt.

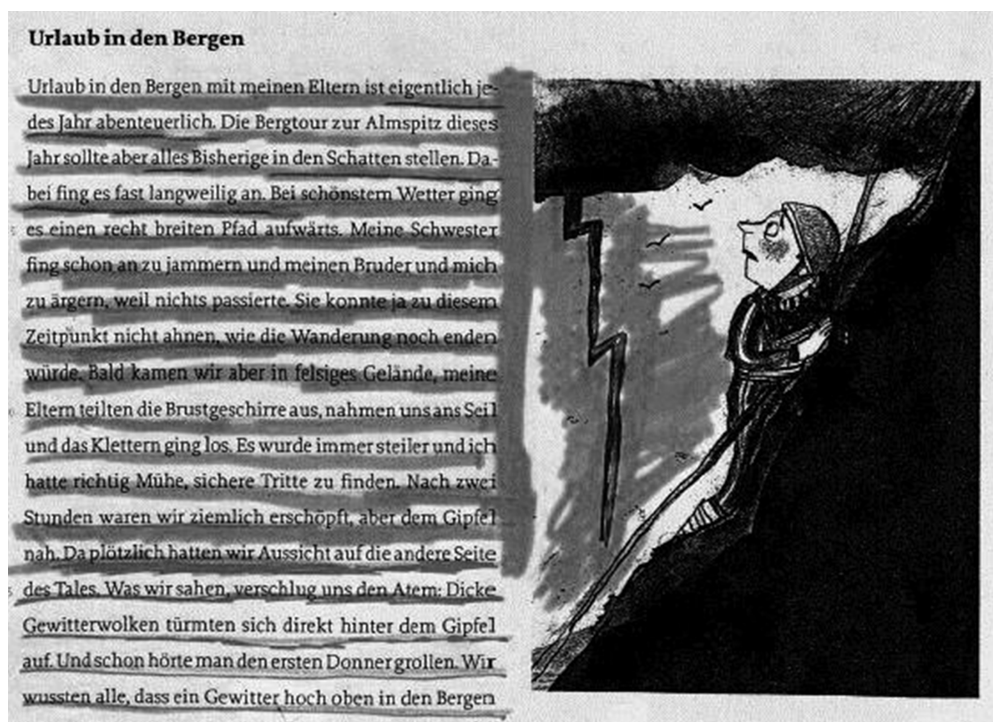


Beispiel 4. 02-1-DU-02-08091523-Einleitung

108 leh was spricht denn daFÜR; =

$109=$ dass hier die einleitung noch ! NICHT! zu ende ist

[in zeile neun; (2.9)]

110 kev [((meldet sich)) ]

111 leh KEvin,

112 kev hier ähm (.) es gibt ja auch eine SCHLINGe (.) sozusagen-=

$113=$ dass man nicht sofort ANfängt äh (-) die gesch

(. ) die geSCHICHte ab (.) ab hm zu (xxx) äh (-) verRAten; (.)

114 da sind sie erst mal bei dem geSCHIRR auspacken;

115 und [da ]

116 leh [geNAU: ; ]

117 kev fängt ja nicht (.) das (-) der !HAUPT! teil an sozusAgen;

118 leh [RICHtig; ]

119 kev [oder ] was SPANnendes;

120 leh geNAU.=ne, (.)

121 denn der au (. ) EIgentliche hauptteilsatz ist ja dann da-=

$122 \quad=$ geNAU.

123 wo sie in den in den (. )

|überhaupt KLETtern; I=ne?=

$\mid((1+r$ Handfläche fahren parallel abwärts $)) \mid$

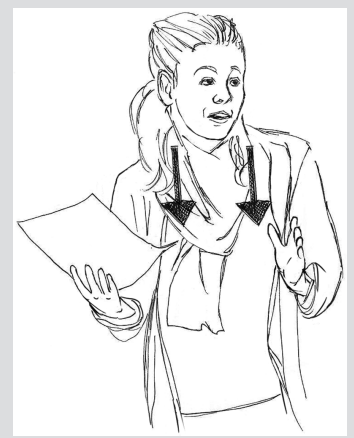

124

=wenn ich noch gar kein

I geSCHIRR hab,

$\mid((1 \mathrm{H}+\mathrm{rH}$ zeichnen $2 \mathrm{x}$ weite $\mathrm{Klammer}$ vor Körper $)) \mid$ 


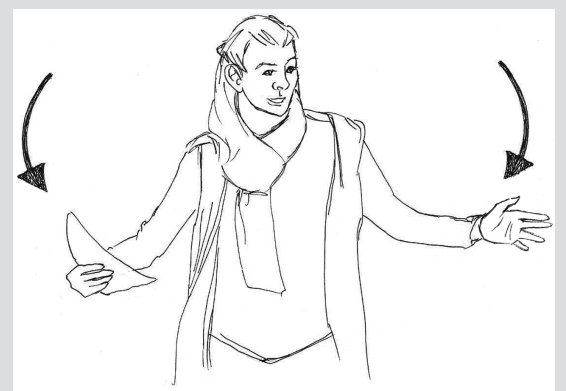

dann kann ich auch noch nicht ANfangen, (.)

IDAS

I ist so_n (.) so_n punkt- (.)

$\mid(($ lH zeigt zu Kev $)) \mid$

wo: (.) so_n |asPEKT | wo man sagen kann;

|((rH: R-vertical)) |

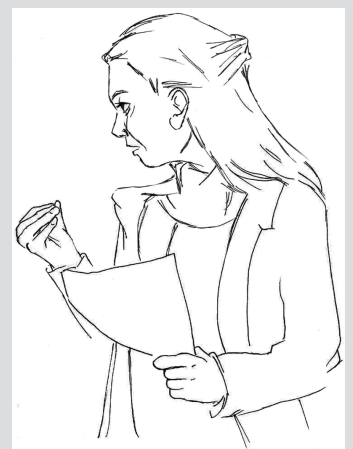

aHA, (.)

jetzt werden die erst AUSgeteilt,

die IVORbereitungen | werden noch getroffen;

$$
\mid((\text { lH zeigt zu Kev })) \mid
$$

(.) ne,

also (.) immer noch kein (.) HAUPTteil, (.)

aber eine weitere ISCHLINge;

$\mid(($ lH zeigt zu Kev $)) \mid$

geNAU; (.)

weil ja etwas (.) ANgedeutet wird; =

=WAS aber noch nicht weiter gefol (. ) verfolgt wird;

JA? (--) 
In einem ersten Schritt deutet Kevin die Textstelle, auf die er mit dem deiktischen „hier“ Bezug nimmt, als Schlinge (Z. 112). Es handelt sich dabei um einen Begriff, der bereits in einer vorangegangenen Stunde als kennzeichnend für Einleitungen etabliert wurde. Nachdem er zunächst erläutert, was unter einer Schlinge zu verstehen ist (Z. 113: „dass man nicht sofort ANfängt [. . .] die geSCHICHte ab (.) ab hm zu [...] verRAten;“), wird die in Frage stehende Textstelle paraphrasiert: „da sind sie erst mal bei dem geSCHIRR auspacken;“ (Z. 114). Mittels solcher explizierender Paraphrasen (Gülich \& Kotschi 1987: 47) erfolgt ein erneuter Blick auf den Text, und zwar aus der Perspektive eines Lesers, der zu diesem eine reflexive Haltung einnehmen kann. Im vorliegenden Fall legt Kevin die Logik der im Text dargestellten Teilhandlung frei: Indem er die zeitliche Situierung (,erst mal“) und das Gewöhnliche des Geschirrauspackens ins Gedächtnis der Beteiligten ruft, plausibilisiert er, dass dieses zum normal course of events (Einleitung) und nicht bereits zum Aufbau von Spannung (Hauptteil) gehört. Schließlich zieht er von der Paraphrase ausgehend eine Folgerung: „da [. . .] fängt ja nicht der !HAUPT!teil an“.

Die Anordnung der Strukturelemente - (1) Deutung, (2) explizierende Paraphrase, (3) Folgerung - ist argumentativ: Es muss gezeigt werden, dass die Textstelle tatsächlich eine Belegfunktion für die Deutung (,Teil der Einleitung') erfüllt. Dies wird mit der explizierenden Paraphrase vorbereitend plausibilisiert und schließlich mit der Folgerung festgestellt. Neben der argumentativen Anordnung der Strukturelemente sind für das Vertextungsverfahren auch bestimmte sprachliche Markierungen konstitutiv. An zwei Stellen verwendet Kevin den Paraphrasen-Indikator (Kotschi 2010: 1343) sozusagen; dieser wird sowohl der Ausgangsdeutung als auch der Folgerung nachgestellt. Zum einen stellt der Indikator eine Beziehung zwischen Deutung und Textstelle her; zum anderen kontextualisiert er den Status der Äußerungen als (nicht ganz sichere) Deutung bzw. Folgerung. Er gibt den Rezipient/innen somit eine Anleitung für das Verstehen der Äußerung: Zwischen Textstelle und Paraphrase soll eine Äquivalenzrelation hergestellt werden. Um die Rezipient/innen in dieser Weise zu instruieren, können Sprecher/innen aus einer Reihe von Paraphrasen-Indikatoren wählen und dabei den Grad der Äquivalenz der Paraphrasenrelation näher qualifizieren. Durch die Wahl des Heckenausdrucks sozusagen wird letztere als eher vage markiert.

Kevins Beitrag wird von der Lehrerin mehrfach bestätigt (Z. 116, 118, 120). Während in Kevins Äußerung die explikative bzw. plausibilisierende Funktion der Paraphrase noch relativ implizit geblieben ist (so verweist er mit der Modalpartikel ja auf geteiltes Wissen, anstatt dieses zu explizieren), liefert die Lehrerin nun eine solche Explizierung nach, die sie mit der Kausalkonjunktion denn 
projiziert. Insofern fungiert ihr Beitrag auch als Modell für die Nutzung des Vertextungsverfahrens:

- Die Deutung der Textpassage als zur Einleitung gehörig wird noch einmal ex negativo (,wo beginnt der Hauptteil?') begründet. Dies erfordert die genaue Referenz auf den „EIgentliche[n] hauptteilsatz“ (Z. 121). Nachdem dieser zunächst gesucht wurde (,wo sie in den in den“), wird die Referenz („überhaupt KLETtern;“) auch mit sichtbaren semiotischen Ressourcen verdeutlicht. Die parallel abwärts geführten Hände markieren bzw. umgrenzen virtuell die Textpassage, mit der der Hauptteil beginnt. Streeck (2011: 142) fasst die Funktion solcher darstellender Gesten deshalb als „marking“ oder „projective indexing“. 8

- Die Deutung erfährt sodann durch Explizieren einer Schlussregel (vgl. 4.2.2) eine Stützung ${ }^{9}$ (Z. 124-125: ,wenn ich noch gar kein geSCHIRR hab, dann kann ich auch noch nicht anfangen“), wobei die Darstellung konkretisierend aus der Ich-Perspektive erfolgt. Auch hier wird die Referenz auf Passagen des Textes gestisch veranschaulicht. Im Unterschied zu der absteckenden Geste in Z. 123 umfassen die Hände nun mit einer ausholenden Bewegung all die vorbereitenden Handlungen, die die Rezipient/innen (die anderer Meinung waren) noch der Einleitung zuschlagen sollen. Die Geste instruiert also die Rezipient/innen, die verbal geäußerten Handlungen (Geschirr auspacken, Vorbereitungen treffen) zu einer Gruppe zusammenzufügen, die die Einleitung ausmachen. Den Gesten ist gemeinsam, dass sie fachliche Operationen des ,Behandelns` von Texten - das genaue Referenzieren auf und Eingrenzen von Passagen - durch Manipulation an einem virtuellen, vorgestellten Text sichtbar machen und veranschaulichen.

- Wie bei Kevin wird die nun folgende explizierende Paraphrase mit einem Paraphrasen-Indikator projiziert: „das ist so_n punkt, [...] so_n asPEKT wo man sagen kann;“ (Z. 126-127). Erstens fällt auf, dass der Indikator nun an seinen eigentlichen Ort, also in unmittelbarer Nähe der Paraphrase platziert wird (im Unterschied zu Kevin, der die Strukturelemente der Deutung und Folgerung mit Paraphrasen-Indikatoren markiert hatte). Zweitens bringt der Indikator im Unterschied zu sozusagen eine größere Genauigkeit des Bezugs zwischen Text und Paraphrase zum Ausdruck. Die Präzision der Referenz wird durch eine gesture of precision grip verdeutlicht, eine

8 Er nennt das Indexieren „projektiv“, weil das Markieren nicht am realen, sondern am imaginierten Gegenstand erfolgt.

9 Das Etablieren und Nutzen einer Schlussregel wird als eigenes Verfahren in Kapitel 4.2.2 thematisiert. Dass es hier eine Komponente eines komplexeren Verfahrens bildet, weist darauf hin, dass Vertextungsverfahren auch kombiniert und ineinander eingebettet werden können. 
Geste, bei der sich Daumen und Zeigefinger berühren und in dieser Haltung zeitlich koordiniert mit prosodisch akzentuierten Elementen der verbalen Äußerung mehrfach abwärts geführt werden („ring-vertical“, vgl. Kendon 2004: 241). Mit der Geste greift die Lehrerin gleichsam einen bestimmten Teil des Textes heraus, um ihn - adressiert an die Vertreter der konträren Deutung - klärend zu behandeln. ${ }^{10}$

- Für die explizierende Paraphrase (re-)inszeniert die Lehrerin die Gedanken beim Lesen; sie spricht also laut aus, "was einem der Text sagt': „aha . . .“ (Z. 128-130). Mit dieser Inszenierung und Vorführung ihres gedanklichen Prozesses zerlegt sie einen ansonsten nicht sichtbaren mentalen Vorgang sequenziell in Schritte und macht diesen intersubjektiv verfügbar und nachvollziehbar. Auch hieran zeigt sich, dass das Vertextungsverfahren auf Anschaulichkeit und Situierung zum Zwecke des Aushandelns und Teilens von Deutungen angelegt ist.

- Die Folgerung ,immer noch kein HAUPTteil . . .“ (Z. 131/132) wird mit also markiert. Abschließend erfolgt eine wiederholende Erklärung, worin die Schlinge besteht (Z. 134/135).

Das Verfahren findet sich in unseren Daten ausschließlich in argumentativen Kontexten, etwa, wenn zu einem Text o. Ä. konkurrierende Deutungen existieren oder Deutungen explorativ plausibilisiert werden. Seine Funktion besteht darin, Deutungen intersubjektiv nachvollziehbar zu machen. Die für das Vertextungsverfahren konstitutiven Strukturelemente umfassen (1) eine Deutung, (2) eine explizierende Paraphrase, die eine Inszenierung des gedanklichen Prozesses einschließen kann, und (3) eine Folgerung. Es handelt sich somit um ein mehrteiliges Verfahren, bei dem eigene Gedanken in begründender Weise mit dem Text verwoben werden. Dies erfordert eine genaue Referenz auf Textstellen, die auch mittels Gesten markiert und ,herausgegriffen' werden können. Verbal wird die Paraphrase mit Indikatoren wie sozusagen, das ist . . . wo man sagen kann, das ist projiziert.

Im Unterschied zum Verdichten zu einer Kernaussage erfolgt beim explizierenden Paraphrasieren keine Reduktion auf das Wesentliche, sondern eine an das Alltagswissen anknüpfende, situierende und veranschaulichende Entfaltung, was zumeist mit der Produktion eines längeren Äußerungspakets einhergeht. Beide Verfahren dürften aber eine zentrale Rolle in „Auslegungskulturen“ (Assmann 1992: 93) spie-

10 Damit bestätigt sich Kendons Beobachtung: "R[ing]-gestures are brought into play whenever, for the speaker, 'making precise' or clarification seems necessary or important in gaining the agreement, the conviction or the understanding of the interlocutor" (2004: 241). 
len, in denen Texte als Träger des kulturellen Gedächtnisses Institutionen der Interpretation und Praktiken des Deutens verlangen.

\subsubsection{Etablieren und Nutzen einer Schlussregel}

Das Etablieren einer Schlussregel beinhaltet, dass die Beteiligten aus Beispielen allgemeine Sätze bzw. Präsuppositionen erschließen, die in der weiteren Argumentation als Schlussregel dienen. Kienpointner (1992) unterscheidet zwischen schlussregelbenützenden und schlussregeletablierenden Argumentationsschemata. In unseren Daten zeigt sich jedoch, dass beide oft unmittelbar aufeinander folgend genutzt werden.

Das Etablieren einer Schlussregel lässt sich in informellen Gesprächen vergleichsweise selten beobachten. Hier bleibt die Schlussregel meist implizit, weil die Beteiligten im Kontext des Gesprächs als (im ethnomethodologischen Sinne) ,unproblematisch` bzw. geteilt voraussetzen, auf welchen regelhaften Zusammenhang sie sich jeweils beziehen. In fachlichen Gesprächen dagegen erweist sich das Etablieren einer Schlussregel als ein Verfahren, mit dem allgemeingültiges Wissen generiert und zugleich abgesichert werden kann. Es handelt sich in gewisser Weise um ein selbstreflexives Verfahren, mit dem sich die Beteiligten der Grundlagen ihres argumentativen Schließens vergewissern. Insofern findet es sich in unseren Daten grundsätzlich in Kontexten explorativen Argumentierens, in denen bis dato selbstverständliche Annahmen problematisiert oder offene Fragen gestellt werden. In Beispiel 5 geschieht dies auf folgende Weise: Im Mathematikunterricht (Thema: Umrechnen von Einheiten, s. Beispiel 2) hatte Doro den Schluss gezogen, dass 2800 Krill benötigt werden, um die Länge eines 28 Meter langen Wals zu erhalten. Im Mathematikbuch war angegeben, dass ein Krill einen Zentimeter lang ist.

Beispiel 5. 02-5-MU-05-08150952-Krill

Tafel:

\begin{tabular}{lr}
\hline & Hund \\
& $100 \mathrm{~cm}$ \\
\hline Krill & \\
$1 \mathrm{~cm}$ &
\end{tabular}


001 dor der krill ist jaEInen zentimeter; (.)

002 und wenn ich jetzt DREItausend zentimeter raus hab,

003 ist das ja für mich dreiTAUsend. (-)

004 aber wenn (-) der BLAUwal (.) in dem buch steht ja der ist ACHTundzwanzig meter;

005 und wenn man das dann umrechnet in zentimeter,

006 dann sind zwei (. ) tausendachthundert.

$007 \quad$ (2.5)

008 leh STIMMT das was doro (rechnet)?

009 sus ((murmeln))

010 leh lasst uns mal an doros überlegung RAN.

011 wie viel krill brauch ich denn für eine MAUS? (---)

012 für die LÄNGE einer maus?

013 sus ((melden sich))

014 leh ((blickt zu Fab))

015 fab ZEHN;

016 leh wie viel krill brauch ich denn für die länge eines HUNdes?

017 sus ((melden sich))

018 leh ((blickt zu Pek))

019 pek HUNdert;

020 leh wie viel krill brauch ich für die länge eines ZAUnes;

$021 \quad(5.2)$

$022 \quad$ ((nickt Mia zu))

023 mia EINtausend,

024 leh (-) wie viel krill brauch ich für dreißig MEter;

$((.)$.

030 leh |woHER

| ((stellt Handflächen einander gegenüber $)) \mid$

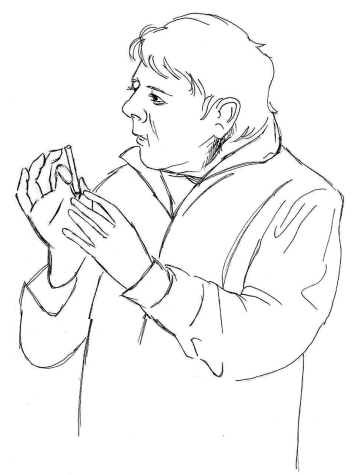


wusste denn- (---)

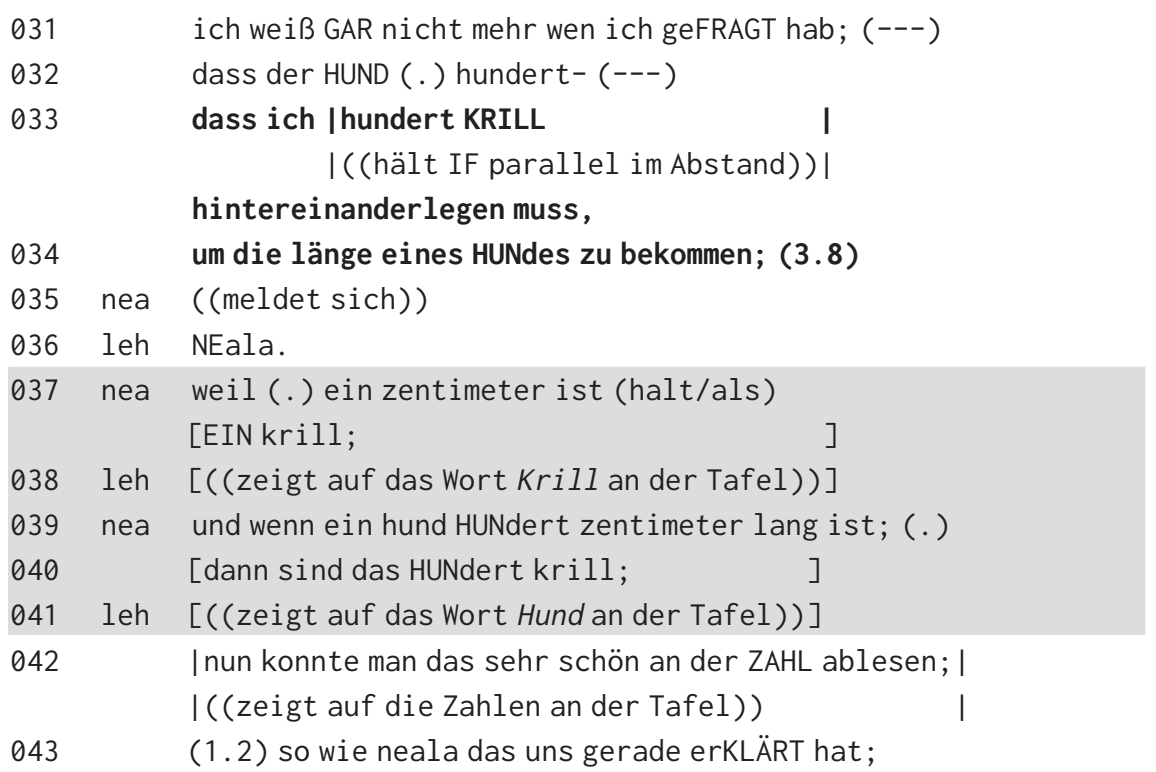

Die Vorbereitung für das Etablieren einer Schlussregel wird durch den Lehrer gesteuert. Nachdem Doro - eine nicht explizierte Schlussregel nutzend - ihre Lösung vorgetragen hat (Z. 1-6), stellt der Lehrer zunächst deren Richtigkeit in Frage (Z. 8). Als keine Meldungen seitens der Schülerinnen und Schüler erfolgen (Z. 9), verlangsamt er die thematische Progression, indem er metakommunikativ expliziert, woran als nächstes gemeinsam kommunikativ gearbeitet werden soll: „lasst uns mal an doros überlegung RAN.“ Er stellt nun eine Reihe von Fragen (Z. 11-24), die die Schülerinnen und Schüler zum Umrechnen beispielhafter Längen in Krill veranlassen. Wenngleich die lokalen Fragen im Gewand unterrichtstypischer Prüffragen daherkommen, hat doch die metakommunikative Projektion (Z. 10) Hinweise darauf gegeben, dass die Beispielumrechnungen nicht zum Zweck des Übens oder Testens mathematischer Fertigkeiten erfolgen, sondern als Methode zu verstehen sind, die es den Beteiligten ermöglicht, sich kollaborativ über die Grundlage von Doros „überlegung“ klar zu werden.

Dass ein neuer Schritt auf dem Weg der kollaborativen Klärung initiiert wird, kontextualisiert der Lehrer zunächst mit einer diskursstrukturierenden und metapragmatischen Geste (Streeck 2009: 179): Indem er seine Handflächen in engem Abstand einander gegenüberstellt, zeichnet er den Übergang vom Breiten zum Engen vor: Das Durchgehen einer Vielzahl von Beispielen wird nun durch das Konstruieren von etwas Spezifischem, Eingrenzbarem abgelöst. Überlappend mit der Geste produziert er das „woHER“ und lässt es durch die 
Pause zunächst für sich stehen; auf diese Weise lenkt er die Aufmerksamkeit auf die thematische Einengung und erzeugt Spannung. Die woher-Frage (Z. 30, 35-36) etabliert also einen Zugzwang zur Explikation des den gerade vollzogenen Beispiellösungen zugrundeliegenden Schlussverfahrens. Dabei werden zentrale Informationen aus dem Vorangegangenen noch einmal mit einer veranschaulichenden Geste verdeutlicht. Retrospektiv wird hier zugleich ein induktives Vorgehen erkennbar, bei dem eine Schlussregel zunächst an Beispielen genutzt wird, bevor sie dann auf der Grundlage dieser vollzogenen Praxis thematisiert und expliziert wird.

Auf das „woher“ mit „weil“ antwortend, nennt Neala zunächst die zentrale Grundlage ihres Schlusses (den allgemeinen Satz oder mit Toulmin gesprochen den „warrant“): „weil (.) ein zentimeter ist (halt/als) EIN krill;“(Z. 38). Sie stellt dann, ganz im Sinne von Toulmins (1958) Basis-Argumentationsschema, eine regelhafte Beziehung zwischen dem Datum (der Hund ist $100 \mathrm{~cm}$ lang) und der Folgerung (man benötigt hundert Krill für die Länge eines Hundes) her. Auf diese Weise formuliert sie eine wenn-dann-Aussage mit zwei durch ein logisches und verknüpften Bedingungen: Wenn es wahr ist, dass der Hund einhundert Zentimeter lang ist, und wenn außerdem wahr ist, dass ein Krill einen Zentimeter misst, dann entspricht die Länge eines Hundes der von einhundert Krill. Damit ist nun eine generische Schlussregel expliziert, mit der auch andere Fälle zu lösen sind: Anstelle des Platzhalters „Hund“ können nun alternative Daten eingesetzt werden. Genau dies geschieht im Folgenden.

Ausdruckseitig erfolgt die Explikation der Schlussregel mittels eines Konditionalgefüges (Z. 40-41). Die Protasis gibt mit der Konjunktion wenn die Bedingung an, unter der die in der Apodosis formulierte Konsequenz (eingeleitet mit dann) gilt. Zeitlich koordiniert mit Nealas Explikation zeigt der Lehrer auf die entsprechenden Elemente an der Tafel. Er macht die Komponenten der Schlussregel damit auch visuell für die anderen Schülerinnen und Schüler nachvollziehbar.

Ähnlich wie beim Formulieren einer Kernaussage liegt hier ein Vertextungsverfahren vor, das mit einer Kondensierung auf das Wesentliche und einer satzförmigen Formulierung einhergeht. Seine zentrale Funktion besteht darin, regelhafte Beziehungen bzw. Gesetzmäßigkeiten zu explizieren. Dies erlaubt zum einen eine Ablösung vom konkreten Einzelfall. Ist eine Schlussregel einmal etabliert, so lässt sie sich wiederum für die Lösung beliebig vieler vergleichbarer Fälle nutzen. Zum anderen ebnet das Vertextungsverfahren den Weg für die methodisch reflektierte Konstruktion von Wissen und ist damit für Lehr-Lernprozesse hochgradig funktional. 


\subsection{Modi des Vertextens: Situieren vs. Abstrahieren}

Der folgende Abschnitt nimmt nun die in 4.1 und 4.2 beschriebenen Vertextungsverfahren erneut in den Blick und betrachtet sie im Hinblick auf ihre Verfahrensweise. Die Analysen haben bereits durchscheinen lassen, dass zwischen den Vertextungsverfahren über die Gattungsgrenzen hinweg Gemeinsamkeiten und Unterschiede im Hinblick auf grundlegende Modi des Vertextens bestehen.

Den Verfahren Beispiel geben (B1), schrittweises Vorführen eines Vorgehens (B2) und explizierendes Paraphrasieren von Belegen für eine Deutung (B4) liegen folgende Gemeinsamkeiten bzgl. der Verfahrensweise zugrunde:

- Komplexe - der Beobachtung nur eingeschränkt zugängliche - Handlungen (mathematische und textbezogene Operationen oder Gedankenfolgen) werden in Teilschritte ,entpackt', chronologisiert und ggf. segmentiert.

- Dies hat i.d.R. die Produktion längerer Äußerungspakete zur Folge.

- Durch Zeigen, gestisches Darstellen, schrittweises Vorführen von Teilschritten, Redewiedergaben bzw. das Inszenieren gedanklicher Prozesse werden fachliche Operationen - Addieren, Umrechnen, Einleiten oder Eingrenzen von Textstrukturen - sinnlich wahrnehmbar und damit im wortwörtlichen Sinne anschaulich gemacht.

- Die Rezipient/innen werden als Zuschauer/innen adressiert; die gemeinsame Aktivität wird als Vorführung kontextualisiert.

- Ausdruckseitig erfordern die Vertextungsverfahren komplexe Konfigurationen semiotischer Ressourcen. Dazu gehören Mittel zur Markierung der zeitlichen Reihung (und dann, erst mal), prosodische Mittel zur Segmentierung sowie Mittel, die eine genaue Referenz auf den Text (die Gleichung an der Tafel, die Geschichte) ermöglichen: Zeigen, gestisches Markieren/Indexieren, Lokaladverbien oder metasprachliche Umschreibungen wie das is so_n punkt wo man sagen kann, im HAUPTteil da.

Ich nenne den Modus, dem die drei Vertextungsverfahren folgen, in Anlehnung an Brünner \& Gülich (2002) Situieren. Die Sprecherin führt einer noch nicht verstehenden oder noch zweifelnden Zuhörerschaft einen fachlichen Zusammenhang vor Augen, indem sie ihn in einem raumzeitlichen Bezugsrahmen situiert. Es überrascht nicht, dass diese ,Kontextanreicherung ${ }^{611}$ zumeist durch für das Erzählen typische Verfahren wie bspw. Chronologisierung und animierte Rede bewerkstelligt wird. Zudem wird durch Herstellung eines Alltagsbezugs an geteiltes Wissen angeknüpft und dieses aktualisiert (Brünner \& Gülich 2002: 81f).

11 Gemeint ist hier der im Äußerungspaket dargestellte Kontext. 
Das schrittweise Vorführen von Handlungen bzw. gedanklichen Prozessen ist darauf ausgerichtet, dass die Zuhörer/innen eine Vorstellung (Redder 2016: 310) entwickeln. Dies ermöglicht erstens die Bearbeitbarkeit einzelner Wissenskomponenten, indem diese bspw. herausgegriffen und korrigiert oder expliziert werden (Harren 2015: 157). Zweitens zielt das Veranschaulichen darauf, den Rezipient/innen einen komplexen Zusammenhang verständlich zu machen. Generiert wird hier ein Wissen, das an Erfahrung anknüpft und dieses bislang implizite oder „stillschweigende Wissen“ in „artikuliertes Wissen“ (Polanyi 1966) bspw. über fachliche Handlungsweisen transformiert.

Demgegenüber verfahren das Verdichten zu einer Kernaussage (B3) und das Etablieren und Nutzen einer Schlussregel (B5) nach einem gänzlich anderen Modus des Vertextens:

- Beide Verfahren zeichnen sich dadurch aus, dass eine Ablösung von konkreten, beispielhaften Fällen erfolgt: Die situativen, zeitlichen, personellen Details der Handlung des Theaterstücks oder einer Reihe beispielhafter Umrechnungen werden ausgeblendet.

- Die Sprecher/innen stellen keinen persönlichen Bezug zum Dargestellten her und adressieren ihre Rezipient/innen als Allgemeinheit.

- Die Vertextung ist auf Komprimierung und Verknappung ausgerichtet. Es entstehen z. T. satzförmige Äußerungspakete.

- Ausdrucksseitig werden vorgeformte Ausdrücke (es geht darum, dass), Konditionalgefüge und Indefinitpronomen verwendet, mit denen die fall-/ kontextübergreifende Gültigkeit des Geäußerten zum Ausdruck gebracht wird. Auf gestische Ressourcen wird größtenteils verzichtet.

Den Vertextungsmodus, dem die Verfahren folgen, nenne ich Abstrahieren. Letzteres wird dabei weniger als kognitiver Akt, sondern als kommunikative Praktik verstanden (vgl. Deppermann 2011: 119), mit der das ,Wesentliche“ eines Sachverhalts in sequenziell organisierten Schritten herausgearbeitet wird. Im Unterschied zu der für den Modus des Situierens typischen Kontextanreicherung und Veranschaulichung erfolgt beim Abstrahieren eine Ablösung vom Einzelfall. Mittels Ausblenden situativer Details und Herausarbeiten des Wesentlichen wird Wissen einer Konservierung bzw. Aufbewahrung (Luckmann 1995/2002: 193) und nicht, wie beim Situieren, einer Bearbeitung zugeführt. Für die verknappende Formulierung werden häufig vorgeprägte Ausdrücke und sprachliche Mittel des Generalisierens genutzt. Die Vertextungsverfahren sind somit darauf ausgerichtet, transsituativ gültiges Wissen zu generieren, zu nutzen und/oder argumentativ abzusichern und in einen logischsystematischen Bezugsrahmen einzuordnen. Die ökonomische und formelhafte Form ermöglicht dabei erstens die Memorierbarkeit, zweitens die Übertragbarkeit des Wissens auf andere Fälle und drittens die präzise Bezugnahme auf das for- 
mulierte Wissen in späteren Situationen. Insofern verwundert es nicht, dass kaum gestische Mittel eingesetzt werden, da diese die Transportabilität und erneute Bezugnahme verkomplizieren würden.

Es ist somit festzuhalten, dass die beschriebenen Modi des Vertextens auf unterschiedliche Zwecke - das Bearbeitbar- und Verständlichmachen auf der einen Seite und das konservierende Formulieren und Übertragbarmachen auf der anderen Seite - zugeschnitten sind. Gleichwohl sollten die Modi nicht als dichotom, sondern eher als Pole eines Kontinuums aufgefasst werden, auf dem sich Vertextungsverfahren als mehr oder wenig stark situierend oder abstrahierend verorten lassen. Hervorzuheben ist, dass beide Modi für Prozesse der Wissensgenerierung und -transmission hochgradig funktional sind.

Tabelle 1 zeigt sämtliche auf Basis unseres Korpus rekonstruierten Vertextungsverfahren nach Gattungen und Modi gruppiert. Mit wenigen Ausnahmen sind die Vertextungsverfahren gattungsspezifisch.

\section{Zusammenfassung und Forschungsdesiderate}

Ziel dieses Beitrags war es, Vertextungsverfahren des Erklärens und Argumentierens in fachlichen Unterrichtsgesprächen $\mathrm{zu}$ beschreiben und auf diesem Wege die Sprachlichkeit fachlicher Erkenntnis- und Lernprozesse genauer zu fassen. Mit Blick auf die für den Unterricht zentralen diskursiven Praktiken des Erklärens und Argumentierens hat der gesprächsanalytische Zugriff nachgezeichnet, wie Erklärer bzw. Proponenten/Opponenten mit Vertextungsverfahren komplexe fachliche Zusammenhänge her- und darstellen. Dabei hat sich gezeigt, dass sich Erklärungen und Argumente je nach kontextueller Anforderung mit einer ganzen Reihe unterschiedlicher Vertextungsverfahren hervorbringen lassen. Letztere zeichnen sich durch unterschiedliche Weisen des Entpackens bzw. Zusammenschnürens von Wissenselementen aus und verlangen unterschiedliche contextual configurations (Goodwin 2013) lexikalischer, syntaktischer, prosodischer und gestischer Ressourcen. Auch hat sich gezeigt, dass Vertextungsverfahren z. T. kombiniert und ineinander eingebettet werden können.

Die Analyse hat weiterhin zutage gefördert, dass die beschriebenen Vertextungsverfahren zwei grundlegend unterschiedlichen Modi zugeordnet werden können: Einige der explanativen und argumentativen Verfahren operieren im Modus des Situierens, andere im Modus des Abstrahierens. Beide Modi erfüllen im Rahmen der Wissenskonstruktion und -vermittlung distinkte epistemische Funktionen. Während situierende Vertextungsverfahren dazu dienen, komplexe und der direkten Beobachtung nur schwer zugängliche Handlungen und Zusammenhänge ver- 


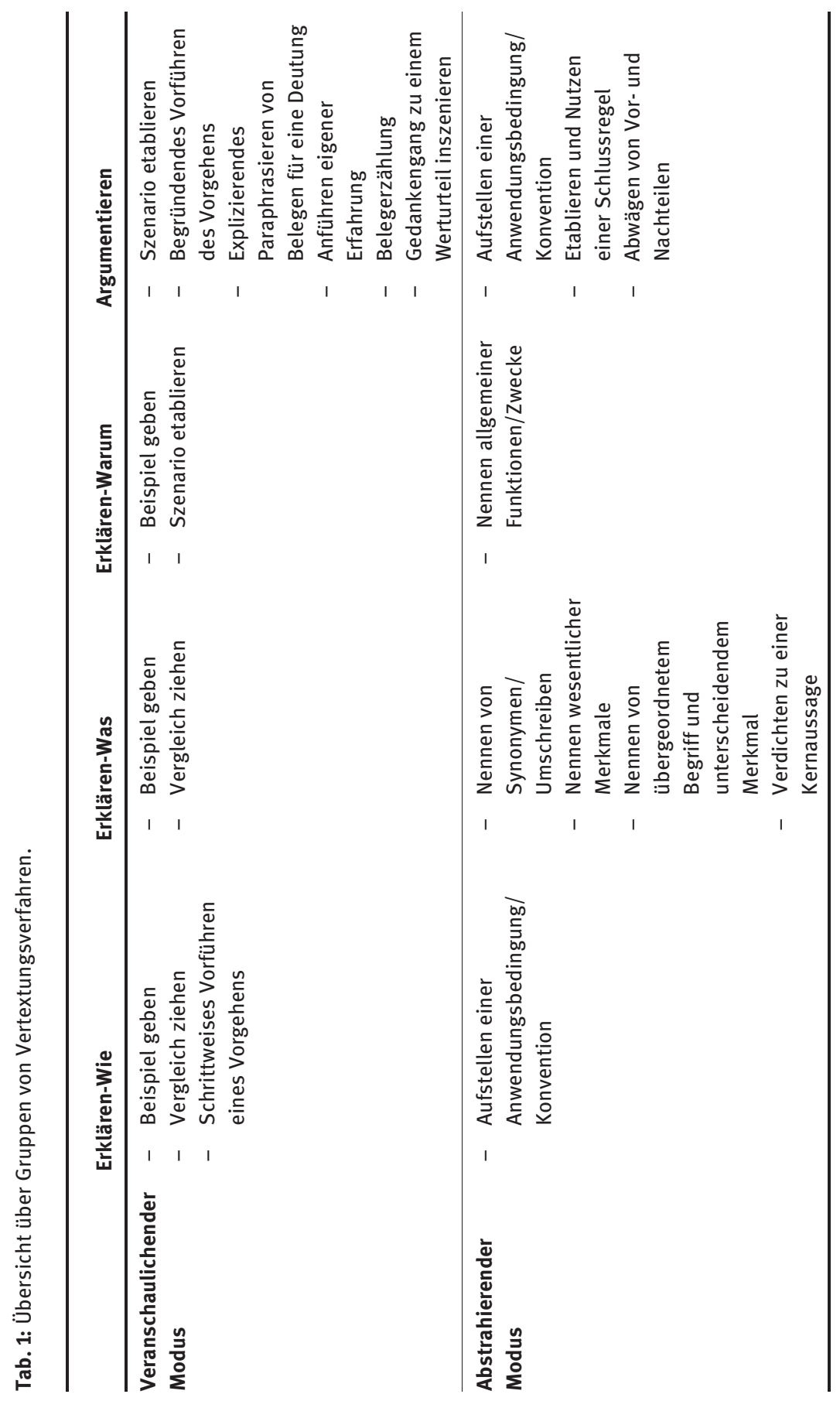


ständlich und bearbeitbar zu machen, ist der abstrahierende Modus darauf zugeschnitten, transsituativ gültiges Wissen zu generieren, und zwar in einer kondensierten Form, die die Reproduzierbarkeit, Übertragbarkeit und spätere präzise Bezugnahme erlaubt. Vertextungsverfahren erweisen sich damit tatsächlich als wesentlich für die Konstruktion, Absicherung und Weitergabe fachlichen Wissens. Darüber hinaus lässt sich über die gesprächsanalytische Rekonstruktion von Vertextungsverfahren auch die Emergenz ganz unterschiedlicher Wissenstypen (Knoblauch 2008; Janich \& Birkner 2015) nachzeichnen: das Vorgangs- bzw. Prozesswissen einerseits und das in einen logisch-systematischen Bezugsrahmen eingeordnete Wissen andererseits. In beiden Fällen handelt es sich um ,artikuliertes Wissen“ (Polanyi 1966), um Wissen also, das explizit beschrieben werden kann und der Reflexion zugänglich ist.

In sprachdidaktischer Perspektive lassen sich nun auch sprachlich-diskursive Anforderungen ableiten, die die Darstellung komplexer fachlicher Zusammenhänge an Lernende stellt. Die Darstellung erfordert erstens ein breites Repertoire von Vertextungsverfahren, über die je nach Modus des Vertextens unterschiedliche Funktionen realisiert werden. Zweitens gilt es im Vollzug von Unterrichtsgesprächen nicht nur online zu kontextualisieren, in welche diskursive Praktik (Erklären oder Argumentieren) ein Zugzwang steuert, sondern auch zu erkennen, ob im jeweiligen sequenziellen Kontext eher situierend oder eher abstrahierend erklärt oder begründet werden soll. Die Annahme, Bildungssprache sei grundsätzlich konzeptionell schriftlich geprägt, erweist sich demzufolge als ergänzungsbedürftig. Sowohl kontextanreichernde als auch kontextabstrahierende Verfahren sind für Prozesse der Wissenskonstruktion funktional und haben ihren Platz im Fachunterricht. Während die situierenden Verfahren die z. T. komplexe Konfiguration räumlicher, materialer, verbaler und verkörpernder semiotischer Ressourcen erfordern, beruhen abstrahierende Verfahren auf einer Reduktion auf das Wesentliche und einer vordringlich explizitsprachlichen Darstellung.

Mit Blick auf die empirische Erforschung von Vertextungsverfahren und ihrer Aneignung eröffnen sich weitere Fragen, die hier abschließend kurz skizziert werden sollen. Hinsichtlich der Kontextsensitivität wäre genauer zu klären, ob sich in unterschiedlichen Kontexten der Wissenskommunikation systematisch unterschiedliche Vorkommen von Vertextungsverfahren beobachten lassen (vgl. bspw. Wyßuwa \& Beier 2013 zu Beispielen und Beispielerzählungen in der Weiterbildung und im Schulunterricht), die wiederum zur Konstitution des jeweiligen Kontextes beitragen. Daran schließt sich auch die Frage an, ob Vertextungsverfahren für die Belange unterschiedlicher Fächer spezialisiert sind. Bezogen auf Unterricht wäre des Weiteren zu untersuchen, ob situierende und abstrahierende Vertextungsverfahren in systematisch unterschiedlichen sequenziellen Kontexten (bspw. ,Erarbeiten“ vs. ,Sichern`) verortet sind. 
Aus der Perspektive der Aneignung stellt sich die Frage, ob der Erwerb von Vertextungsverfahren einer bestimmten Logik folgt (erst situierend, dann abstrahierend?) und wie der Erwerb von Vertextungsverfahren mit dem von sprachstrukturellen Fähigkeiten zusammenhängt. Von ebenso zentraler sprachdidaktischer Bedeutung sind Studien zu den Ressourcen des Erwerbs: Werden Vertextungsverfahren vorrangig implizit und beiläufig erworben oder auch über Modelle und explizite Instruktion? Lassen sich gar Transfereffekte von der Arbeit an der schriftlichen Vertextung auf mündliche Vertextungsverfahren feststellen (vgl. zu umgekehrten Transfereffekten Domenech \& Krah in diesem Band)?

Die Reihe der Fragen lässt vermuten, dass mit den Verfahren und Modi des Vertextens eine Ebene bildungssprachlicher Praktiken ins Visier geraten ist, deren weitere Untersuchung Aufschluss darüber $\mathrm{zu}$ geben verspricht, wie Wissen und unterschiedliche Wissenstypen sprachlich und interaktiv erzeugt werden. Zum anderen ist mit ihnen einer der Ansatzpunkte lokalisiert, an dem sich die sprachlichdiskursiven Anforderungen genauer beschreiben lassen, die fachliche Wissenskonstruktionsprozesse für Lernende mit unterschiedlichen Diskurserwerbsressourcen beinhalten.

\section{Literatur}

Assmann, Jan (1992): Das kulturelle Gedächtnis. Schrift, Erinnerung und politische Identität in frühen Hochkulturen. München: Beck.

Ayaß, Ruth (2013): Form und Funktion kategorischer Formulierungen. In Jörg Bergmann, Thomas Luckmann \& Ruth Ayaß (Hrsg.), Kommunikative Konstruktion von Moral. Band 1: Struktur und Dynamik der Formen moralischer Kommunikation, 106-124. Opladen: Westdeutscher Verlag.

Becker-Mrotzek, Michael (1989): Schüler erzählen aus ihrer Schulzeit. Eine diskursanalytische Untersuchung über das Institutionswissen. Frankfurt a.M.: Peter Lang.

Behrens, Ulrike \& Olaf Gätje (Hrsg.) (2016): Mündliches und schriftliches Handeln im Deutschunterricht. Wie Themen entfaltet werden. Frankfurt a.M.: Peter Lang.

Bergmann, Jörg \& Uta Quasthoff (2010): Interaktive Verfahren der Wissensgenerierung: Methodische Problemfelder. In Ulrich Dausendschön-Gay, Christine Domke \& Sören Ohlhus (Hrsg.), Wissen in (Inter-)Aktion. Verfahren der Wissensgenerierung in unterschiedlichen Praxisfeldern, 21-34. Berlin, New York: De Gruyter.

Birkner, Karin \& Oliver Ehmer (Hrsg.) (2013): Veranschaulichungsverfahren im Gespräch. Mannheim: Verlag für Gesprächsforschung.

Brinker, Klaus \& Jörg Hagemann (2000): Themenstruktur und Themenentfaltung in Gesprächen. In Klaus Brinker (Hrsg.), Text- und Gesprächslinguistik. Ein internationales Handbuch zeitgenössischer Forschung. Band 2, 1252-1263. Berlin: De Gruyter.

Brinker, Klaus (1992): Linguistische Textanalyse. 3. Aufl. Berlin: Erich Schmidt Verlag. Brünner, Gisela (2013): Vermittlungsstrategien in Gesundheitssendungen: Die Rolle von Metaphern, Vergleichen und anderen Verfahren der Veranschaulichung. In Karin Birkner 
\& Oliver Ehmer (Hrsg.), Veranschaulichungsverfahren im Gespräch, 18-43. Mannheim: Verlag für Gesprächsforschung.

Brünner, Gisela \& Elisabeth Gülich (2002): Verfahren der Veranschaulichung in der ExpertenLaien-Kommunikation. In Gisela Brünner \& Elisabeth Gülich (Hrsg.), Krankheit verstehen. Interdisziplinäre Beiträge zur Sprache in Krankheitsdarstellungen, 17-93. Bielefeld: Aisthesis Verlag.

Caffi, Claudia \& Klaus Hölker (1995): Examples. Introduction. Versus. Quaderni di studi semiotici 70/71, 3-17.

Clark, Herbert \& Richard Gerrig (1990): Quotations as Demonstrations. Language in Society 66 (4), 764-805.

Czicza, Dániel (2015): Konstruktionen in der Wissenschaftssprache. In Jörg Bücker, Susanne Günthner \& Wolfgang Imo (Hrsg.), Konstruktionen im Spannungsfeld von sequenziellen Mustern, kommunikativen Gattungen und Textsorten, 81-106. Tübingen: Stauffenburg.

Dausendschön-Gay, Ulrich, Christine Domke \& Sören Ohlhus (2010): Einleitung „Wissen in (Inter-)Aktion“. In Ulrich Dausendschön-Gay, Christine Domke \& Sören Ohlhus (Hrsg.), Wissen in (Inter-)Aktion. Verfahren der Wissensgenerierung in unterschiedlichen Praxisfeldern, 1-19. Berlin, New York: De Gruyter.

Dausendschön-Gay, Ulrich, Elisabeth Gülich \& Ulrich Krafft (2007): Vorgeformtheit als Ressource im konversationellen Formulierungs- und Verständigungsprozess. In Heiko Hausendorf (Hrsg.), Gespräch als Prozess. Linguistische Aspekte der Zeitlichkeit verbaler Interaktion, 181-219. Tübingen: Narr Verlag.

Deppermann, Arnulf (2007): Grammatik und Semantik aus gesprächsanalytischer Sicht. Berlin: De Gruyter.

Deppermann, Arnulf (2011): The Study of Formulations as a Key to an Interactional Semantics. Human Studies 34, 115-128.

Deppermann, Arnulf (2015): Wissen im Gespräch: Voraussetzung und Produkt, Gegenstand und Ressource. InLiSt (57), 1-31.

Deppermann, Arnulf \& Reinhold Schmitt (2008): Verstehensdokumentationen: Zur Phänomenologie von Verstehen in der Interaktion. Deutsche Sprache 36 (3), 220-245.

Drescher, Martina (1992): Verallgemeinerungen als Verfahren der Textkonstitution. Untersuchungen zu französisichen Texten aus mündlicher und schriftlicher Kommunikation. Stuttgart: Franz Steiner Verlag.

Ehlich, Konrad (2014): Argumentieren als sprachliche Ressource des diskursiven Lernens. In Antonie Hornung, Gabriella Carobbio \& Daniela Sorrentino (Hrsg.), Diskursive und textuelle Strukturen in der Hochschuldidaktik. Deutsch und Italienisch im Vergleich, 41-54. Münster: Waxmann.

Ehmer, Oliver (2011): Imagination und Animation. Die Herstellung mentaler Räume durch animierte Rede. Berlin, New York: De Gruyter.

Ehmer, Oliver (2013): Veranschaulichungsverfahren im Gespräch. In Karin Birkner \& Oliver Ehmer (Hrsg.), Veranschaulichungsverfahren im Gespräch, 2-17. Mannheim: Verlag für Gesprächsforschung.

Feilke, Helmuth (2012): Was sind Textroutinen? In Helmuth Feilke \& Katrin Lehnen (Hrsg.), Schreib- und Textroutinen. Theorie, Erwerb und didaktisch-mediale Modellierung, 1-31. Frankfurt a.M.: Peter Lang.

Feilke, Helmuth (2014): Argumente für eine Didaktik der Textprozeduren. In Thomas Bachmann \& Helmuth Feilke (Hrsg.), Werkzeuge des Schreibens. Theorie und Potentiale einer Didaktik der Textprozeduren, 11-34. Stuttgart: Fillibach bei Klett. 
Garfinkel, Harold (1967): Studies in ethnomethodology. Cambridge: Polity Press.

Goodwin, Charles (1995): Sentence Construction Within Interaction. In Uta Quasthoff (Hrsg.), Aspects of oral communication, 198-219. Berlin: De Gruyter.

Goodwin, Charles (2013): The co-operative, transformative organization of human action and knowledge. Journal of Pragmatics 46 (1), 8-23.

Grundler, Elke \& Sara Rezat (2016): Argumentative Themenentfaltung in Schülergesprächen und Schülertexten. In Olaf Gätje \& Ulrike Behrens (Hrsg.), Mündliches und schriftliches Handeln im Deutschunterricht. Wie Themen entfaltet werden, 149-168. Frankfurt a.M.: Peter Lang.

Grundler, Elke \& Rüdiger Vogt (Hrsg.) (2006): Argumentieren in Schule und Hochschule: interdisziplinäre Studien. Tübingen: Stauffenburg.

Gülich, Elisabeth \& Heiko Hausendorf (2000): Vertextungsmuster Narration. In Klaus Brinker et al. (Hrsg.), Text- und Gesprächslinguistik. Ein internationales Handbuch zeitgenössischer Forschung, 369-385. Berlin: De Gruyter.

Gülich, Elisabeth \& Thomas Kotschi (1983): Partikeln und Paraphrasen-Indikatoren (am Beispiel des Französischen). In Harald Weydt (Hrsg.), Partikeln und Interaktion, 249-262. Tübingen: Niemeyer.

Gülich, Elisabeth \& Thomas Kotschi (1987): Reformulierungshandlungen als Mittel der Textkonstitution. Untersuchungen zu französischen Texten aus mündlicher Kommunikation. In Wolfgang Motsch (Hrsg.), Satz, Text, sprachliche Handlung, 199-261. Berlin: Akademie-Verlag.

Gülich, Elisabeth \& Thomas Kotschi (1996): Textherstellungsverfahren in mündlicher Kommunikation. Ein Beitrag am Beispiel des Französischen. In Wolfgang Motsch (Hrsg.), Ebenen der Textstruktur. Sprachliche und kommunikative Prinzipien, 37-80. Tübingen: Niemeyer.

Günthner, Susanne (1995a): Gattungen in der sozialen Praxis. Die Analyse „kommunikativer Gattungen“ als Textsorten mündlicher Kommunikation. Deutsche Sprache (3), 193-218.

Günthner, Susanne (1995b): Exemplary stories: the cooperative construction of moral indignation. Versus. Quaderni di studi semiotici 70/71, 147-175.

Günthner, Susanne (2006): Von Konstruktionen zu kommunikativen Gattungen: Die Relevanz sedimentierter Muster für die Ausführung kommunikativer Aufgaben. gidi Arbeitspapierreihe (1), 1-21.

Handro, Saskia (2013): Sprache und historisches Lernen. Dimensionen eines Schlüsselproblems des Geschichtsunterrichts. In Michael Becker-Mrotzek, Karen Schramm, Eike Thürmann \& Johannes Vollmer (Hrsg.), Sprache im Fach - Sprachlichkeit und fachliches Lernen, 317-333. Münster: Waxmann.

Harren, Inga (2015): Fachliche Inhalte sprachlich ausdrücken lernen. Sprachliche Hürden und interaktive Vermittlungsverfahren im naturwissenschaftlichen Unterrichtsgespräch in der Mittel- und Oberstufe. Mannheim: Verlag für Gesprächsforschung.

Hausendorf, Heiko (2011): Kunstkommunikation. In Stephan Habscheid (Hrsg.), Textsorten, Handlungsmuster, Oberflächen: Linguistische Typologien der Kommunikation, 509-535. Berlin: De Gruyter.

Hausendorf, Heiko (2016). Kunst. In Ludwig Jäger, Werner Holly, Peter Krapp, Samuel M. Weber \& Simone Heekeren (Hrsg.), Sprache - Kultur - Kommunikation. Ein internationales Handbuch zu Linguistik als Kulturwissenschaft, 496-505. Berlin, Boston: De Gruyter Mouton. 
Hausendorf, Heiko \& Uta Quasthoff (2005): Sprachentwicklung und Interaktion. Eine linguistische Studie zum Erwerb von Diskursfähigkeiten. Radolfzell: Verlag für Gesprächsforschung. http://www.verlag-gespraechsforschung.de/2005/quasthoff.htm

Heinemann, Wolfgang (2000a): Textsorte - Textmuster - Texttyp. In Klaus Brinker et al. (Hrsg.), Text- und Gesprächslinguistik. Ein internationales Handbuch zeitgenössischer Forschung. Band 1, 507-523. Berlin: De Gruyter.

Heinemann, Wolfgang (2000b): Vertextungsmuster Deskription. In Klaus Brinker et al. (Hrsg.), Text- und Gesprächslinguistik. Ein internationales Handbuch zeitgenössischer Forschung. Band 1, 356-369. Berlin: De Gruyter.

Heller, Vivien (2012): Kommunikative Erfahrungen von Kindern in Familie und Unterricht. Passungen und Divergenzen. Tübingen: Stauffenburg.

Heller, Vivien (2016): Meanings at hand: coordinating semiotic resources in explaining mathematical terms in classroom discourse. Classroom Discourse 7 (3), 253-275.

Heller, Vivien (2017a): Managing knowledge claims in classroom discourse. The public construction of a homogeneous epistemic status. Classroom Discourse 8 (2), 156-174.

Heller, Vivien (2017b): Lerngelegenheiten für Erklären und Argumentieren: Wie partizipieren mehrsprachige Schülerinnen und Schüler an bildungssprachlichen Praktiken? In Isabel Fuchs, Stephan Jeuk \& Werner Knapp (Hrsg.), Mehrsprachigkeit: Spracherwerb, Unterrichtsprozesse, Schulentwicklung, 165-182. Stuttgart: Fillibach bei Klett.

Heller, Vivien \& Miriam Morek (2015): Academic discourse as situated practice: An introduction. Linguistics \& Education 31, 174-186.

Heller, Vivien, Uta Quasthoff, Susanne Prediger \& Anna Vogler (2017): Bildungssprachliche Praktiken aus professioneller Sicht: Wie deuten Lehrende Schülererklärungen und -begründungen? In Bernt Ahrenholz \& Claudia Schmellentin (Hrsg.), Fachunterricht und Sprache in schulischen Lehr-/Lernprozessen, 139-160. Tübingen: Narr.

Hempel, Carl-Gustav \& Paul Oppenheim (1948): Studies in the Logic of Explanation. Philosophy of Science 15, 135-175.

Heritage, John \& Rodney D. Watson (1980): Aspects of the properties of formulations in natural conversations: Some instances analysed. Semiotica 30 (3/4), 245-262.

Hoffmann, Ludger (2006): Fachsprache/Language of Specific Purposes. In Ulrich Ammon (Hrsg.), Sociolinguistics. An international handbook of the science of language and society, Volume 1, 232-238. Berlin: De Gruyter.

Janich, Nina \& Karin Birkner (2015): Text und Gespräch. In Ekkehard Felder \& Andreas Gardt (Hrsg.), Handbuch Sprache und Wissen, 195-220. Berlin: De Gruyter.

Kääntä, Leila, Gabriele Kasper \& Arja Piirainen-Marsh (2018): Explaining Hooke's Law: Definitional Practices in a CLIL Physics Classroom. Applied Linguistics 39 (5), 694-717.

Kärkkäinen, Elise (2006): Stance taking in conversation. From subjectivity to intersubjectivity. Text \& Talk 26 (6), 699-731.

Kendon, Adam (2004): Gesture. Visible action as utterance. Cambridge, New York: Cambridge University Press.

Keppler, Angela (1989): Schritt für Schritt. Das Verfahren alläglicher Belehrungen. Soziale Welt 40 (4), 538-556.

Kern, Friederike (2003): Bedeutung und Interaktion: Spielerklärung bei Kindern. In Stefanie Haberzettl \& Heide Wegener (Hrsg.), Spracherwerb und Konzeptualisierung, 257-273. Frankfurt a.M.: Peter Lang.

Kesselheim, Wolfgang (2010): Wissenskommunikation multimodal: Wie Museumsbesucher sich über eine Museumsvitrine verständigen. Fachsprache (3-4), 122-144. 
Klein, Josef (2001): Erklären und Argumentieren als interaktive Gesprächsstrukturen. In Klaus Brinker et al. (Hrsg.), Text- und Gesprächslinguistik. Ein internationales Handbuch zeitgenössischer Forschung. Band 2, 1309-1329. Berlin: De Gruyter.

Klein, Wolfgang \& Juliane von Stutterheim (1992): Textstruktur und referentielle Bewegung. Zeitschrift für Literaturwissenschaft und Linguistik (LiLi) 86, 67-92.

Knoblauch, Hubert (2008): Sinnformen, Wissenstypen und Kommunikation. In Herbert Willems (Hrsg.), Lehr(er)buch Soziologie. Band 1, 131-146. Wiesbaden: VS Verlag.

Knorr-Cetina, Karin (1984): Die Fabrikation von Erkenntnis. Zur Anthropologie der Naturwissenschaft. Frankfurt a.M.: Suhrkamp.

Knorr-Cetina, Karin (1991): Epistemic Cultures: Forms of Reason in Science. History of Political Economy 23 (1), 105-122.

König, Katharina (2013): Generalisieren, Moralisieren: Redewiedergabe in narrativen Interviews als Veranschaulichungsverfahren zur Wissensübermittlung. In Karin Birkner \& Oliver Ehmer (Hrsg.), Veranschaulichungsverfahren im Gespräch, 201-223. Mannheim: Verlag für Gesprächsforschung.

Komor, Anna (2013): Semantische Fähigkeiten und ihre Aneignung im Vor- und Grundschulalter - am Beispiel der Begriffserklärung im Unterricht. In Stefan Jeuk \& Ingrid Schmid-Barkow (Hrsg.), Differenzen diagnostizieren und Kompetenzen fördern im Deutschunterricht, 43-58. Stuttgart: Fillibach bei Klett.

Kuhn, Leema \& Reiser, Brian (2009): Making sense of argumentation and explanation. Science Education 93 (1), 26-55.

Labov, William \& Joshua Waletzky (1967): Narrative Analysis. Oral Versions of Personal Experience. In: June Helm (Hrsg.), Essays on the verbal and visual arts, 12-44. Seattle: University of Washington Press.

Latour, Bruno \& Steve Woolgar (1986). Laboratory life. The construction of scientific facts. Princeton, N.J: Princeton University Press.

Linz, Erika (2018): Transkriptive Praktiken der Kunstkommunikation. In Jan Gerwinski, Stephan Habscheid \& Erika Linz (Hrsg.), Theater im Gespräch, 203-234. Berlin: De Gruyter.

Luckmann, Thomas (2002): Der kommunikative Aufbau der sozialen Welt und die Sozialwissenschaften. In Thomas Luckmann \& Hubert Knoblauch (Hrsg.), Wissen und Gesellschaft. Ausgewählte Aufsätze 1981 - 2002, 157-182. Konstanz: UVK.

Lymer, Gustav, Jonas Ivarsson, Hans Rystedt, Ase Johnsson, Sara Asplund \& Magnus Bath (2014): Situated abstraction. From the particular to the general in second-order diagnostic work. Discourse Studies 16 (2), 185-215.

Marx, Nicole \& Torsten Steinhoff (2017): Das „TraBi“-Projekt: Förderung der mündlichen Bildungssprache in den Sachfächern. BiSS-Journal 7, 33-35.

McNeill, David (1992): Hand and mind. What gestures reveal about thought. Chicago: University of Chicago Press.

Morek, Miriam (2012). Kinder erklären. Interaktionen in Familie und Unterricht. Tübingen: Stauffenburg.

Morek, Miriam (2016). Formen mündlicher Darstellung in situ: Zur Komplexität von Diskursanforderungen in Unterrichtsgesprächen. In Olaf Gätje \& Ulrike Behrens (Hrsg.), Mündliches und schriftliches Handeln im Deutschunterricht. Wie Themen entfaltet werden, 95-132. Frankfurt a.M.: Peter Lang.

Morek, Miriam, Vivien Heller (2012). Bildungssprache - kommunikative, epistemische, soziale und interaktive Aspekte ihres Gebrauchs. Zeitschrift für Angewandte Linguistik, 57, 67-101. 
Morek, Miriam, Vivien Heller \& Uta Quasthoff (2017): Argumentieren und Erklären. Konzepte, Modellierungen und empirische Befunde im Rahmen der linguistischen Erwerbs- und Unterrichtsforschung. In Iris Meissner \& Eva Wyss (Hrsg.), Erklären und Argumentieren. Konzepte und Modellierungen in der Angewandten Linguistik, 11-45. Tübingen: Stauffenburg.

Müller, Frank \& Aldo Di Luzio (1995): Stories as examples in everyday argument. Versus (70/71), 115-145.

Oberzaucher, Frank \& Ulrich Dausendschön-Gay (2014): Kategorisieren. In Jörg Bergmann, Ulrich Dausendschön-Gay \& Frank Oberzaucher (Hrsg.), „Der Fall“. Studien zur epistemischen Praxis professionellen Handelns, 345-379. Bielefeld: transcript.

Ohlhus, Sören (2014): Erzählen als Prozess. Interaktive Organisation und narrative Verfahren in mündlichen Erzählungen von Grundschulkindern. Tübingen: Stauffenburg.

Ohlhus, Sören (2016): Narrative Verfahren und mediale Bedingungen. Überlegungen zur Analyse mündlicher und schriftlicher Erzählungen. In Ulrike Behrens \& Olaf Gätje (Hrsg.), Mündliches und schriftliches Handeln im Deutschunterricht. Wie Themen entfaltet werden, 39-65. Frankfurt a.M.: Peter Lang.

Polanyi, Michael (1966): The tacit dimension. New York: University of Chicago Press.

Quasthoff, Uta M. (2012): Globale und lokale Praktiken in unterschiedlichen diskursiven Genres: Wie lösen L2-Sprecher globale Anforderungen bei eingeschränkter sprachstruktureller Kompetenz im Deutschen? In Heike Roll \& Andrea Schilling (Hrsg.), Mehrsprachiges Handeln im Fokus von Linguistik und Didaktik, 47-65. Duisburg: Universitätsverlag Rhein-Ruhr.

Quasthoff, Uta \& Dietrich Hartmann (1982): Bedeutungserklärungen als empirischer Zugang zu Wortbedeutungen. Zur Entscheidbarkeit zwischen holistischen und komponentiellen Bedeutungskonzeptionen. Deutsche Sprache (2), 97-118.

Quasthoff, Uta, Vivien Heller \& Miriam Morek (2017): On the sequential organization and genre-orientation of discourse units in interaction. An analytic framework. Discourse Studies 19 (1), 84-110.

Raible, Wolfgang (1995): Arten des Kommentierens - Arten der Sinnbildung - Arten des Verstehens. In Jan Assmann (Hrsg.), Text und Kommentar, 51-73. München: Fink.

Redder, Angelika (2016): Theoretische Grundlagen der Wissenskonstruktion im Diskurs. In Jörg Kilian, Birgit Brouër \& Dina Lüttenberg (Hrsg.), Handbuch Sprache in der Bildung, 297-318. Berlin, Boston: De Gruyter.

Rehbein, Jochen (1982): Worterklärungen türkischer Kinder. Osnabrücker Beiträge zur Sprachtheorie: OBST 22, 122-157.

Rettich, Heike (2011): Beispielkommunikation. Formen und Funktionen der Beispielverwendung in der verbalen Interaktion. Universität Koblenz-Landau, Koblenz-Landau.

Roelcke, Thorsten (2010): Fachsprachen. 3. neu bearbeitete Auflage. Berlin: Erich Schmidt. Sacks, Harvey (1995): Lectures on conversation. Cambridge, Mass: Blackwell.

Sacks, Harvey \& Harold Garfinkel (1970): On formal structures of practical action. In Edward Tiryakian \& John McKinney (Hrsg.), Theoretical Sociology. Perspectives and Developments, 338-366. New York: Appleton-Century-Crofts.

Schegloff, Emanuel (1996): Turn organization: one intersection of grammar and interaction. In Elinor Ochs, Emanuel Schegloff \& Sandra Thompson (Hrsg.), Interaction and grammar, 52-133. Cambridge, New York: Cambridge University Press.

Schlinkmann, Eva (2018): Rekonstruktive Verfahren. In Jan Gerwinski, Stephan Habscheid \& Erika Linz (Hrsg.), Theater im Gespräch, 301-371. Berlin: De Gruyter. 
Schwitalla, Johannes (1991): Das Illustrieren - eine narrative Textsorte mit zwei Varianten. In Hugo Steger, Jürgen Dittmann, Hannes Kästner \& Johannes Schwitalla (Hrsg.), Erscheinungsformen der deutschen Sprache. Literatursprache, Alltagssprache, Gruppensprache, Fachsprache, 189-204. Berlin: Schmidt.

Schwitalla, Johannes (2006): Gesprochenes Deutsch. Eine Einführung. 3. Auflage Berlin: Schmidt.

Stivers, Tanya, Lorenza Mondada \& Jakob Steensig (2011): Knowledge, morality and affiliation in social interaction. In Tanya Stivers, Lorenza Mondada \& Jakob Steensig (Hrsg.), The morality of knowledgment in Conversation, 3-24. Cambridge: Cambridge University Press.

Streeck, Jürgen (2009): Gesturecraft. The manu-facture of meaning. Amsterdam: John Benjamins.

Stude, Juliane (2005): Mündliche und schriftliche Spielerklärungen von Grundschulkindern. Erste Analysen des OLDER-Korpus. Dortmund (Projektpaper Nr. 3).

Stutterheim, Christiane von \& Wolfgang Klein (2008): Mündliche Textproduktion: Informationsorganisation in Texten. In Nina Janich (Hrsg.): Textlinguistik: 15 Einführungen, 217-235. Tübingen: Narr.

Toulmin, Stephen (1958): The Uses of Argument. Cambridge: Cambridge University Press.

Trautmann, Caroline (2004): Argumentieren. Funktional-pragmatische Analysen praktischer und wissenschaftlicher Diskurse. Frankfurt am Main: Lang.

Wyßuwa, Franziska \& Frank Beier (2013): Beispielerzählungen und Szenarioentwicklung in der Weiterbildung als Veranschaulichungen von Wissen und Relevanzen. In Karin Birkner \& Oliver Ehmer (Hrsg.), Veranschaulichungsverfahren im Gespräch, 133-155. Mannheim: Verlag für Gesprächsforschung. 